\title{
Functional characterization of water transport and cellular localization of three aquaporin paralogs in the salmonid intestine
}

\section{Steffen S. Madsen ${ }^{1}$, Jesper H. Olesen ${ }^{1}$, Konstanze Bedal ${ }^{1}$, Morten Buch Engelund $^{1}$, Yohana M. Velasco-Santamaría ${ }^{1}$ and Christian K. Tipsmark ${ }^{2}$}

1 Institute of Biology, University of Southern Denmark, Odense, Denmark

${ }^{2}$ Department of Biological Sciences, University of Arkansas, Fayetteville, NC, USA

\section{Edited by:}

Shigehisa Hirose, Tokyo Institute of Technology, Japan

\section{Reviewed by:}

Carlos M. Luquet, Centro de Ecologia Aplicada del Neuquen, Argentina Martin Grosell, University of Miami, USA

Roderick Nigel Finn, University of Bergen, Norway

*Correspondence:

Steffen S. Madsen, Institute of Biology, University of Southern

Denmark, Campusvej 55, DK-5230

Odense M, Denmark.

e-mail:steffen@biology.sdu.dk
Intestinal water absorption is greatly enhanced in salmonids upon acclimation from freshwater (FW) to seawater (SW); however, the molecular mechanism for water transport is unknown. We conducted a pharmacological characterization of water absorption in the rainbow trout intestine along with an investigation of the distribution and cellular localization of three aquaporins (Aqp1aa, -1ab, and -8ab) in pyloric caeca, middle (M), and posterior (P) intestine of the Atlantic salmon. In vitro iso-osmotic water absorption $\left(J_{v}\right)$ was higher in SW than FW-trout and was inhibited by $\left(\mathrm{mmol} \mathrm{L}^{-1}\right)$ : $0.1 \mathrm{KCN}(41 \%), 0.1$ ouabain (72\%), and 0.1 bumetanide $(82 \%)$ suggesting that active transport, $\mathrm{Na}^{+}, \mathrm{K}^{+}$-ATPase and $\mathrm{Na}^{+}, \mathrm{K}^{+}$, $2 \mathrm{Cl}^{-}$-co-transport are involved in establishing the driving gradient for water transport. $J_{V}$ was also inhibited by $1 \mathrm{mmol} \mathrm{L}^{-1} \mathrm{HgCl}_{2}$, serosally ( $23 \%$ in $\mathrm{M}$ and $44 \%$ in $\mathrm{P}$ ), mucosally $(27 \%$ in $\mathrm{M})$, or both (61\% in $\mathrm{M}$ and $58 \%$ in $\mathrm{P})$, suggesting involvement of both apical and basolateral aquaporins in water transport. The inhibition was antagonized by $5 \mathrm{mmol} \mathrm{L}^{-1}$ mercaptoethanol. By comparison, $10 \mathrm{mmol} \mathrm{L}^{-1}$ mucosal tetraethylammonium, an inhibitor of certain aquaporins, inhibited $J_{V}$ by $20 \%$. In the presence of glucose, mucosal addition of phloridzin inhibited water transport by $20 \%$, suggesting that water transport is partially linked to the $\mathrm{Na}^{+}$-glucose co-transporter. Using polyclonal antibodies against salmon Aqp1aa, -1ab, and -8ab, we detected Aqp1aa, and -1ab immunoreactivity in the brush border and sub-apical region of enterocytes in all intestinal segments. The Aqp8ab antibody showed a particularly strong immunoreaction in the brush border and sub-apical region of enterocytes throughout the intestine and also stained lateral membranes and peri-nuclear regions though at lower intensity. The present localization of three aquaporins in both apical and lateral membranes of salmonid enterocytes facilitates a model for transcellular water transport in the intestine of SW-acclimated salmonids.

Keywords: aquaporin, osmoregulation, salmon, intestine, enterocyte, water absorption

\section{INTRODUCTION}

Animals living in a dehydrating environment need compensatory mechanisms in order to maintain osmotic homeostasis. Terrestrial animals largely overcome this challenge by drinking water followed by intestinal absorption. Accordingly, the intestine in these animals is developed in order to absorb quite large amounts of fluid. Per day the intestine of a human individual absorbs ca. Two liter of ingested water in addition to approximately $7 \mathrm{~L}$ of digestive juice (Ma and Verkman, 1999). Among aquatic animals, marine fishes use one of two strategies to avoid dehydration. Cartilaginous species actively build up the internal osmotic pressure by means of organic osmolytes to slightly exceed the external osmotic pressure. Thereby they may gain water passively across the gill epithelium. Teleost fishes, whether marine or euryhaline, maintain hypo-osmotic body fluids by drinking seawater (SW) and actively excreting surplus ions. It has been known since the classical paper by Smith (1932) that they swallow an appreciable amount of water (drinking rates typically in the range of $1-5 \mathrm{~mL}^{-1} \mathrm{~kg}^{-1} \mathrm{~h}^{-1}$,
Perrott et al., 1992), which is processed and partially absorbed in the gastrointestinal tract (GIT). Water absorption is basically solute linked as proposed in the original standing gradient model by Diamond and Bossert (1967). Several details are known with regard to the processing of imbibed SW along the length of the intestine, in particular concerning the accompanying transport of dissolved ions (see Grosell, 2011). In short, the fluid is desalinated in the esophagus and anterior parts of the GIT by absorption of the major monovalent ions $\mathrm{Na}^{+}$and $\mathrm{Cl}^{-}$. While being moved peristaltically through the more posterior parts, monovalent ions are actively absorbed by enterocytes via a mechanism involving basolateral $\mathrm{Na}^{+}, \mathrm{K}^{+}$-ATPase and cystic fibrosis transmembrane conductance regulator (CFTR)-like $\mathrm{Cl}^{-}$-channels and apical $\mathrm{Na}^{+}$, $\mathrm{Cl}^{-}$and $\mathrm{Na}^{+}, \mathrm{K}^{+}, 2 \mathrm{Cl}^{-}$-co-transporters as well as $\mathrm{HCO}_{3}^{-} / \mathrm{Cl}^{-}$ exchangers. Across the basolateral membrane, $\mathrm{Na}^{+}$ions may be recycled as shown in the toad small intestine by Nedergaard et al. (1999). Divalent ions such as $\mathrm{Mg}^{2+}$ and $\mathrm{Ca}^{2+}$ are largely untouched and precipitate with bicarbonate and sulfate ions due 
to the secretion of bicarbonate and successive osmotic removal of water (Grosell, 2011). Precipitation leads to a reduced osmotic pressure and thus eases the osmotic absorption further.

Whereas the molecular mechanism of salt transport in the GIT is known in some detail, the exact pathway by which water follows is not well established. Theoretically, it may occur via paracellular or transcellular pathways or a combination thereof (Laforenza et al., 2005). Fish and mammalian intestinal epithelia are generally considered as being leaky, thus allowing some paracellular passage of water (Loretz, 1995; Ma and Verkman, 1999). In mammals, the rectum on the other hand has higher transepithelial resistance (TER) and thus a tighter epithelium with low paracellular water permeability (Ma and Verkman, 1999). Sundell et al. (2003) found an increase in the intestinal iso-osmotic fluid uptake rate in vitro $\left(J_{v}\right)$ during SW-acclimation of Atlantic salmon, Salmo salar. This was associated with an increase in TER probably caused by a tightening of the junctions between the enterocytes, and suggests that the increased water absorption occurs via a transcellular rather than paracellular pathway in SW-acclimated salmon.

Transcellular water flux may involve three different pathways: passive diffusion across the lipid bilayers, co-transport with ions and nutrients, and diffusion through aquaporins in the membranes. Water transport across biological membranes was long considered to occur by simple diffusion through the lipid bilayer. But the discovery of the first aquaporin by Preston et al. (1992) showed that water transport across membranes may indeed be greatly enhanced by insertion of molecular water channels. Furthermore, trafficking of aquaporins to and from membranes makes a powerful mechanism to regulate epithelial and cellular water flux both in the short and long term (Gradilone et al., 2003; Valenti et al., 2005; Tingaud-Sequeira et al., 2008). In mammals, it has been shown that the majority of intestinal water absorption occurs through aquaporins present in both apical and basolateral membranes of enterocytes. Aquaporins 1, 3, 4, 7, 8, and 10 have all been demonstrated in enterocytes or surrounding associated endothelial tissue in mammals (Ma and Verkman, 1999; Ishibashi et al., 2009). In addition to aquaporins, it has been proposed that substantial amounts (50\%) of water may be transported by the apical $\mathrm{Na}^{+}$-glucose cotransporter (SGLT1; Loo et al., 2002). This requires, however, that glucose is present in the intestinal lumen. The SGLT1 has been demonstrated by immunocytochemistry (ICC) in the rainbow trout enterocytes (Polakof et al., 2010) but its role in water absorption has to our knowledge never been studied in fish. However, since fish can undergo extended periods of starvation without losing homeostatic control of water balance, it is reasonable to speculate that the role may be minor or at least transient.

Our current knowledge about aquaporins in fish is still rather fragmentary and gathered from many different species. In the whole genome of zebrafish, Danio rerio, 10 aquaporin subfamilies are found, some of which have duplicate or triplicate paralogs (reviewed by Cerdà and Finn, 2010). As in mammals, the expression of specific paralogs appears to be related to tissue type. Some paralogs are expressed more generally whereas others are restricted to certain tissues. In teleosts, the following aquaporins have been demonstrated at mRNA level, protein level, or both in various intestinal segments (esophagus, anterior, posterior region, rectum): Aqp1aa/ab (European eel, Anguilla anguilla: Martinez et al., 2005a; Japanese eel, A. japonica: Aoki et al., 2003; Kim et al., 2010; European seabass, Dicentrarchus labrax: Giffard-Mena et al., 2007; Atlantic salmon, S. salar: Tipsmark et al., 2010; seabream, Sparus aurata: Raldúa et al., 2008), Aqp3 (European eel: Cutler et al., 2007; European seabass: Giffard-Mena et al., 2007; Atlantic salmon: Tipsmark et al., 2010); Aqp4, -7, -8aa (zebrafish: TingaudSequeira et al., 2010), Aqp8ab (zebrafish: Tingaud-Sequeira et al., 2010; Atlantic salmon: Tipsmark et al., 2010), Aqp10 (European eel: Martinez et al., 2005a; Japanese eel: Kim et al., 2010; seabream: Santos et al., 2004; Atlantic salmon: Tipsmark et al., 2010), and Aqp11b and -12 (zebrafish: Tingaud-Sequeira et al., 2010). Only Aqplaa/ab have been localized in enterocytes and thus directly associated with water transport (European eel: Martinez et al., 2005b; Japanese eel: Aoki et al., 2003; seabream: Raldúa et al., 2008). All other aquaporins have either been found in other cell types (mucus cells, lamina propria) or not been localized yet.

On this basis, the present study investigated the mechanism for water transport in the intestine of two salmonids acclimated to SW: rainbow trout, Oncorhynchus mykiss, and Atlantic salmon. We first characterized water transport in isolated intestinal segments in rainbow trout and showed how it was affected by pharmacological agents known to interfere with ion and metabolite transport and aquaporins. Next we developed homologous antibodies against Atlantic salmon aquaporins-1aa, $-1 \mathrm{ab}$, and $-8 \mathrm{ab}$ (formerly Aqp8b in Tipsmark et al., 2010) and investigated their cellular localization by ICC and confocal microscopy. Whereas the intestinal cellular localization of Aqplaa/ab has been investigated in other species, Aqp8ab is a novel paralog in any teleost in this respect.

\section{MATERIALS AND METHODS FISH AND MAINTENANCE}

For characterization of intestinal water transport we used $1^{+}$-yearold rainbow trout (ca. $200 \mathrm{~g}$ ) from an all-female stock obtained from a trout hatchery (Lime Hatchery, Randbol, Denmark). Upon arrival to the Institute of Biology at the University of Southern Denmark (SDU) they were acclimated for at least 4 weeks to $28 \mathrm{ppt}$ re-circulated and biofiltered artificial SW (Red Sea Salt, Eliat, Israel) in $400 \mathrm{~L}$ tanks $\left(14^{\circ} \mathrm{C}, 12: 12\right.$ light:dark period). For immunolocalization studies we used 1-year-old Atlantic salmon (ca. $25 \mathrm{~g}$ ) obtained as pre-smolts from The Danish Centre for Wild Salmon (Randers, Denmark). After spending the spring in outdoor tanks on the SDU Campus, they were transferred to indoor $400 \mathrm{~L}$ tanks in late June with constant temperature and light conditions $\left(14^{\circ} \mathrm{C}, 12: 12\right.$ light:dark). The tanks were supplied with recirculated and biofiltered fresh water. In September, a batch of fish was transferred directly to $25 \mathrm{ppt} \mathrm{SW}\left(14^{\circ} \mathrm{C}\right)$ and acclimated for 3 weeks. All fish were fed $1 \%$ body weight ${ }^{-1}$ per day and food was generally withheld 4 days before an experiment. All experimental protocols were approved by the Danish Animal Experiments Inspectorate and in accordance with the European convention for the protection of vertebrate animals used for experiments and other scientific purposes (\#86/609/EØF).

\section{MEASUREMENT OF ISO-OSMOTIC IN VITRO WATER ABSORPTION $\operatorname{RATE}\left(\boldsymbol{J}_{V}\right)$}

For historical reasons, two slightly different techniques were used to measure water transport capacity under iso-osmotic conditions 
in vitro. The methods were previously cross-validated and gave values in the same range (not shown). In both, the intestine was isolated from a freshly killed rainbow trout and perivisceral fat was carefully removed. The lumen was rinsed with Ringer's solution (in mmol L ${ }^{-1}: 140 \mathrm{NaCl}, 2.5 \mathrm{KCl}, 15 \mathrm{NaHCO}_{3}, 1 \mathrm{KH}_{2} \mathrm{PO}_{4}, 1.5 \mathrm{CaCl}_{2}$, $0.8 \mathrm{MgSO}_{4}, 10$ D-glucose equilibrated with $99 \% \mathrm{O}_{2} / 1 \% \mathrm{CO}_{2}, \mathrm{pH}$ 7.8) and the intestine was transferred to a Petri dish with Ringer's containing $5 \mathrm{mg} \mathrm{L}^{-1}$ of the smooth muscle relaxant papaverine for 10-20 min at $15^{\circ} \mathrm{C}$. In some experiments (ouabain, bumetanide, $\mathrm{KCN}$, mercury) $J_{v}$ was analyzed separately in the middle (light colored) and posterior (darker colored) segments with reference to the ileo-rectal valve (Figure 1A). This was done using the noneverted sac method as described by Collie and Bern (1982). In short, a polyethylene tube (inner diameter $1.1 \mathrm{~mm}$, outer diameter, $1.6 \mathrm{~mm}$ ) with a bulged end was inserted into the anterior end of the segment and fixed by cotton thread. After further rinsing of the lumen with Ringer's, the other end was either tied with cotton thread and the segment was filled with Ringer's through a syringe in the tube. Each sac was carefully checked for leaks before closing the tube with a plastic plug. The samples were pre-incubated for $30 \mathrm{~min}$ in $15^{\circ} \mathrm{C}$ Ringer's solution with $5 \mathrm{mg} \mathrm{L}^{-1}$ papaverine and bubbled with $99 \% \mathrm{O}_{2} / 1 \% \mathrm{CO}_{2}$. After that, the contents were replaced with the test solution and the rate of water transport, $J_{v}$ (in $\mu \mathrm{L} \mathrm{cm}^{-2} \mathrm{~h}^{-1}$ ) was calculated from the decrease of the blotted wet weight of the sacs (linear regression) normalized to surface area of the cut open segment. The weight loss of each sac was monitored every $15 \mathrm{~min}$ for $1.5 \mathrm{~h}$. At the end of an experiment, the surface area was measured by spreading out the opened segment on a glass slide and pressing between two slides with clamps. The outline was marked and transferred to paper with a given weight per square centimeter, cut out, weighed and the area calculated.

In other experiments (phloridzin and tetraethylammonium) the whole (i.e., middle + posterior) intestine was used intact according to the method of Usher et al. (1991). In this method the intestine was held in place in a $500-\mathrm{mL}$ chamber and a $10-\mathrm{cm}$

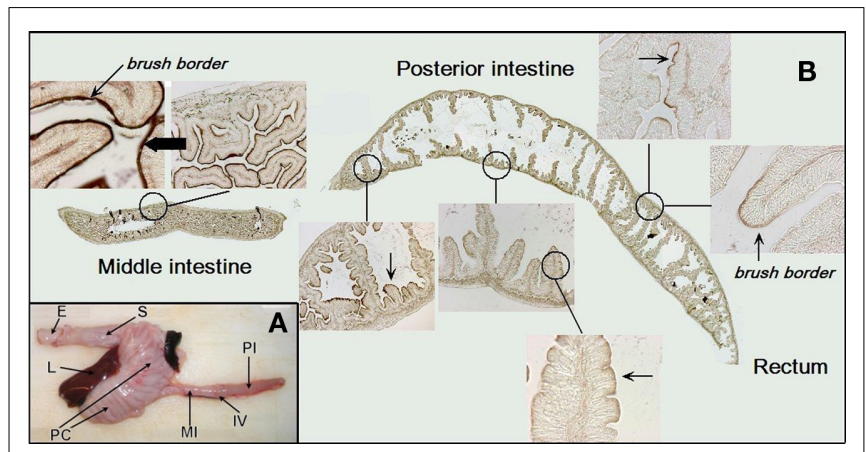

FIGURE 1 | (A) An overview of the morphology of the gastrointestinal tract in Atlantic salmon. (B) A longitudinal section of the entire middle and posterior intestine from SW-acclimated salmon with higher magnifications shown as inserts. The brush border is visualized by staining for alkaline phosphatase activity with NBT/BCIP. Staining of the brush border (dark brown) is seen throughout the intestine, however, more intensely in the middle segment. Abbreviations: E, esophagus; L, liver; S, stomach; PC, pyloric caeca; MI, middle intestine; PI, posterior intestine; IV, ileo-rectal valve. polyethylene tube (same dimensions as above) was inserted into the anterior end connected to a syringe. The posterior end was connected to a $35-\mathrm{cm}$ vertically placed tube. The intestine and tubing were filled with Ringer's and the decrease in the meniscus height was measured every $15 \mathrm{~min}$ for $1 \mathrm{~h}$ (control) and converted into $\mu \mathrm{Lh}^{-1}\left(8.67 \mu \mathrm{L} \mathrm{cm}^{-1}\right.$ tube). The intestine was rinsed every 30 min to prevent mucus accumulation. After $1 \mathrm{~h}$ the Ringer's solution was replaced with the test solution and the measurement was continued for $2.5 \mathrm{~h}$. Control incubation with standard Ringer's was made in a separate series and used for comparison with the test agents. Thus for each preparation $J_{v}$ was measured before and after pharmacological treatment and the effect of the agent was calculated in percent.

\section{EXPERIMENTS}

In different series of experiments the effects of the following pharmacological agents dissolved in Ringer's solution were tested: ouabain $\left(0.5 \mathrm{mmol} \mathrm{L}^{-1}\right.$ serosally; blocks $\mathrm{Na}^{+}, \mathrm{K}^{+}$-ATPase), bumetanide $\left(0.5 \mathrm{mmol} \mathrm{L}^{-1}\right.$ mucosally; blocks $\mathrm{Na}^{+}, \mathrm{K}^{+}, 2 \mathrm{Cl}^{-}$ co-transport), $\mathrm{KCN}\left(0.5 \mathrm{mmol} \mathrm{L}^{-1}\right.$ serosally; metabolic poison), phloridzin ( $1 \mathrm{mmol} \mathrm{L}^{-1}$ mucosally; blocks $\mathrm{Na}^{+}$-glucose cotransport), mercury ( $1 \mathrm{mmol} \mathrm{L}^{-1} \mathrm{HgCl}_{2}$ mucosally, serosally, or both; blocks certain aquaporins), tetraethylammonium (TEA, $10 \mathrm{mmol} \mathrm{L}^{-1}$ mucosally, blocks certain aquaporins). An experiment typically included the following steps after the initial preincubation in Ringer's (30 min): (1) replacement of mucosal solution with fresh Ringer's solution and gravimetric measurement of $J_{v}$ for $1.5 \mathrm{~h}$; (2) replacement of mucosal/serosal solution with test solution (above) and pre-incubation for $30 \mathrm{~min}$; (3) replacement with fresh test solution and gravimetric measurement of $J_{v}$ for $1.5 \mathrm{~h}$. Following the experiments with mercury, the mercury Ringer's was replaced with Ringer's containing $5 \mathrm{mmol} \mathrm{L}^{-1}$ 2-mercaptoethanol in an attempt to antagonize the mercury effect. After a 30-min pre-incubation, the mercaptoethanol Ringer's was replaced with a fresh solution and the $J_{v}$ measured for the next $1.5 \mathrm{~h}$.

\section{ANTIBODIES}

Messenger RNA sequences coding for salmon aquaporins were found in the Atlantic salmon EST (expressed sequence tags) database in GenBank using the following UniGene accession numbers: BT046625 (aqp1aa), BT045044 (aqp1ab), Ssa.15811 (aqp8ab). The $a q p 8 a b$ paralog corresponds to the former $a q p 8 b$ paralog reported by Tipsmark et al. (2010) and was renamed in order to match the nomenclature used in zebrafish (Cerdà and Finn, 2010). The mRNA sequences were translated into protein sequences with the following predicted molecular weights: Aqplaa: $27.3 \mathrm{kDa}$, Aqp1ab: $27.8 \mathrm{kDa}$, Aqp8ab: $27.5 \mathrm{kDa}$. Based on antigenicity plots, the following sequences were used for immunization: Aqplaa: C-terminal residues 243-256: GDYDVNGEETAAVE; Aqp1ab: Cterminal residues 240-253: GPDKENDAPEEGSS; Aqp8ab: Nterminal residues 11-24: GHSTLMSGTKKPTP. Polyclonal antibodies were produced in rabbits by BioGenes (Berlin, Germany). For each antibody the serum preparation was affinity purified and the titer was measured by BioGenes. The affinity purified antibodies were tested in Western blotting and used for immunofluorescence. 


\section{WESTERN BLOTTING ANALYSIS}

For validation of the antibodies homogenates of pyloric caeca were prepared as described below. For Aqplaa a whole-kidney homogenate was used for comparison, since Aqplaa is expressed at higher levels in kidney tubules (Madsen, unpublished data). SW-acclimated salmon were anesthetized in MS222 and killed by decapitation. Pyloric caeca (and kidney tissue) were homogenized in sucrose-EDTA-imidazole buffer (SEI; in $\mathrm{mmol} \mathrm{L}^{-1}: 300$ sucrose, $20 \mathrm{Na}_{2}$ EDTA, 50 imidazol, $\mathrm{pH}$ 7.3) and a cocktail of protease inhibitors (P8340; Sigma-Aldrich, St. Louis, MO, USA) with a Polytron PT 1200 CL for 20 s. The homogenate was centrifuged $10 \mathrm{~min}$ at $2000 \mathrm{~g}$ and the pellet containing whole cells, mitochondria and nuclei was discarded. The supernatant was then centrifuged $40 \mathrm{~min}$ at $60,000 \mathrm{~g}$ at $4^{\circ} \mathrm{C}$ and the membrane fraction and supernatant was separated. The membrane fraction was resuspended in SEI buffer containing 2\% (w/v) sodium deoxycholate and the supernatant was added deoxycholate to a concentration of $2 \%(\mathrm{w} / \mathrm{v})$. Supernatant (pyloric caeca) or the membrane fraction (kidney) was used for Western blotting of the individual aquaporins depending on the abundance of the proteins in the fraction. An aliquot of the homogenate was saved for measuring protein concentration using the method described by Lowry et al. (1951). The NuPAGE system (Invitrogen, Carlsbad, CA, USA) was used for electrophoresis and blotting according to manufacturer's protocols. The samples were mixed with reducing sample buffer (NuPAGE ${ }^{\circledR}$ LDS Sample Buffer, Invitrogen) containing 3\% dithiothreitol (Merck chemicals, Darmstadt, Germany) and heated at $70^{\circ} \mathrm{C}$ for $10 \mathrm{~min}$ ensuring reduction of the proteins. The samples were loaded on a $4-12 \%$ gradient Bis-Tris polyacrylamide gel (NuPAGE ${ }^{\circledR} 4-12 \%$ Bis-Tris Gel, Invitrogen) in a volume corresponding to $150 \mu \mathrm{g}$ protein. The electrophoresis was run at $200 \mathrm{~V}$ for $35 \mathrm{~min}$ with MES-running buffer (Invitrogen). The proteins were blotted on a nitrocellulose membrane in a tris-glycine transfer buffer (in mmol L ${ }^{-1}: 7.5$ tris-base, 60 glycine, and 20\% methanol) for $120 \mathrm{~min}$ at $25 \mathrm{~V}$. The membrane was blocked $1 \mathrm{~h}$ in blocking buffer containing $2 \%$ bovine serum albumin (BSA) in TBS-T (in mmol L ${ }^{-1}$ : 20 tris-base, $140 \mathrm{NaCl}, 1 \%$ Tween 20) followed by incubation overnight at $4^{\circ} \mathrm{C}$ with primary antibody (1-5 $\left.\mu \mathrm{g} \mathrm{mL}^{-1}\right)$ in blocking buffer. After four washes in TBS-T, the membrane was incubated with goat anti-rabbit IgG antibody (1:5000) coupled to the fluorescent dye Cy5 (Invitrogen). After four washes and drying overnight, the fluorescence was measured using in a Typhoon Trio Variable mode Imager (GE Healthcare, Little Chalfont, UK) set to the Cy5 excitation and emission wavelengths (649 and $670 \mathrm{~nm}$ respectively). As control for antibody specificity, strips of membranes were incubated with up to 400fold molar excess of the corresponding immunization peptide. These membranes were compared to membranes incubated with the corresponding antibody alone.

\section{GENERAL HISTOLOGICAL PROCEDURES}

Pyloric caeca, anterior and posterior intestines were sampled from SW-acclimated salmon, immediately fixed in paraformaldehyde (4\% PFA, $0.9 \% \mathrm{NaCl}$ in $5 \mathrm{mmol} \mathrm{L}^{-1} \mathrm{NaH}_{2} \mathrm{PO}_{4}$, pH 7.4) overnight at $4^{\circ} \mathrm{C}$. After being washed four times in $70 \%$ ethanol, the tissues were processed through graded series of ethanol and xylene, and finally embedded into paraffin. Serial sections were cut on a
Zeiss Microtome (Microm HM355; Carl Zeiss, Jena, Germany) and placed onto Superfrost Plus glass slides (Thermo Fisher Scientific Inc., Waltham, MA, USA) for subsequent analysis.

\section{OVERVIEW OF THE GROSS MORPHOLOGY OF THE ATLANTIC SALMON INTESTINE}

A deparaffinized $10 \mu \mathrm{m}$ longitudinal section of the entire middle and posterior intestine of a SW salmon was used to visualize the apical brush border. The section was incubated with a combination of $0.4 \mathrm{mmol} \mathrm{L}^{-1}$ 5-bromo-4-chloro-3'-indolyphosphate p-toluidine salt (BCIP) and $0.4 \mathrm{mmol} \mathrm{L}^{-1}$ nitro-blue tetrazolium chloride (NBT) in Tris- $\mathrm{HCl}$ buffer (in $\mathrm{mmol} \mathrm{L}^{-1}$ : 0.12 -amino2-(hydroxymethyl)-1,3-propanediol hydrochloride, $0.1 \mathrm{NaCl}, 10$ $\mathrm{MgCl}_{2}, \mathrm{pH}$ 9.8) for $10 \mathrm{~min}$ which yields an intense, insoluble black-purple precipitate when reacted with alkaline phosphatase (AP). Since AP is a marker enzyme of the intestinal brush border, this method can be used to visualize the presence of microvilli.

\section{IMMUNOFLUORESCENCE LIGHT MICROSCOPY AND CONFOCAL MICROSCOPY}

Microslides with $5 \mu \mathrm{m}$ paraffin sections were placed vertically in an oven at $55^{\circ} \mathrm{C}$ overnight after which they were deparaffinized and demasked by boiling $10 \mathrm{~min}$ in TEG buffer (in $\mathrm{mmol} \mathrm{L}^{-1}$ : 10 tris-base, 1 EGTA, pH 9). Subsequently they were transferred to $50 \mathrm{mmol} \mathrm{L}^{-1} \mathrm{NH}_{4} \mathrm{Cl}$ in phosphate buffered saline (PBS, in mmol L ${ }^{-1}: 137 \mathrm{NaCl}, 2.7 \mathrm{KCl}, 4.3 \mathrm{Na}_{2} \mathrm{HPO}_{4}, 1.4 \mathrm{KH}_{2} \mathrm{PO}_{4}, \mathrm{pH} 7.3$ ) for $30 \mathrm{~min}$ and blocked with $3 \%$ (BSA) in PBS for $30 \mathrm{~min}$ at room temperature. The slides were dual labeled by incubating overnight at $4^{\circ} \mathrm{C}$ with a cocktail (in PBS with $1.5 \%$ BSA) of primary polyclonal rabbit antibodies for one of the aquaporins $\left(1-5 \mu \mathrm{g} \mathrm{mL}^{-1}\right)$ and a monoclonal mouse antibody recognizing all isoforms of the $\mathrm{Na}^{+}, \mathrm{K}^{+}$-ATPase alpha subunit $\left(0.5-1 \mu \mathrm{g} \mathrm{mL}^{-1}\right.$; alpha-5, The Developmental Studies Hybridoma Bank developed under auspices of the NICHD and maintained by The University of Iowa, Department of Biological Sciences, Iowa City, IA, USA). After two washes in PBS at room temperature, the slides were incubated for $2 \mathrm{~h}$ at $37^{\circ} \mathrm{C}$ with a cocktail of two fluorescent secondary antibodies (Alexa Fluor ${ }^{\circledR} 568$ goat anti-rabbit and Oregon Green ${ }^{\circledR} 488$ goat anti mouse IgG, 1:600 dilution; Invitrogen). After two washes in PBS, nuclei were stained with $4^{\prime}$, 6-diamidino-2-phenylindole (DAPI, $0.1 \mu \mathrm{g} \mathrm{mL}^{-1}$ in PBS) for $10 \mathrm{~min}$. Before being sealed with Vectashield (Vector Labs, Burlingame, CA, USA), the slides were washed four times in PBS and two times in milli-Q water. Negative control incubations with 3\% BSA in PBS instead of primary antibodies were made routinely. The fluorescence was inspected on a Leica HC microscope and pictures of representative areas were captured using a Leica DC 200 camera. Confocal images were taken on a Zeiss LSM510 META confocal microscope (Carl Zeiss) using objectives at $63 \times$ and $100 \times$ magnification.

\section{STATISTICAL ANALYSES}

The effect of mercury and mercaptoethanol on $J_{v}$ were evaluated by repeated measures one-way ANOVA followed by a Bonferroniadjusted Fishers least significant differences test $(p<0.05)$. For all other pharmacological agents, the (negative) effects on in vitro water transport capacity $\left(J_{v}\right)$ were evaluated by a paired one-tailed Student's $t$-test with a significance level of $p<0.05$. Statistics were 
carried out using the GraphPad Prism 5.0 software (San Diego, CA, USA).

\section{RESULTS}

\section{OVERALL MORPHOLOGY}

The gastrointestinal system of Atlantic salmon is shown in Figure 1A with the segments involved in water absorption indicated on the figure: the anterior region with numerous pyloric caeca, the middle and posterior segments. The longitudinal section in Figure 1B shows the intensive folding of the luminal epithelium with a clear indication of the apical brush border judged from the positive reaction for AP. The AP reaction is more intense in the middle than in the posterior brush border. The intense brush border is also clearly seen in the transmission electron micrograph in Figure 2, together with other characteristics of enterocytes: apical tight junctions, lateral membrane, several mitochondria, and vesicles in the sub-apical compartment.

\section{VALIDATION OF ANTIBODIES}

The specificity of the affinity purified polyclonal antibodies was validated by Western blotting of tissue homogenates as shown in Figure 3. All three antibodies (Aqplaa, Aqp1ab, and Aqp8ab) detected a protein around $30 \mathrm{kDa}$ in addition to both lower and higher molecular weight bands. For Aqp laa the strongest band was observed around $40 \mathrm{kDa}$. The $30-\mathrm{kDa}$ band matches the expected $\mathrm{Mr}$ of the native aquaporin whereas the higher molecular weight bands may represent various glycosylated forms of the aquaporin. The $30-\mathrm{kDa}$ band and those of higher $\mathrm{Mr}$ disappeared in the neutralization experiment with $400 \times$ molar excess of the immunization peptide.

\section{EFFECT OF PHARMACEUTICALS ON $\boldsymbol{J}_{\boldsymbol{V}}$}

The iso-osmotic water transport rate $\left(J_{v}\right)$ tested in the middle intestine segment was higher in SW than in freshwater (FW) fish

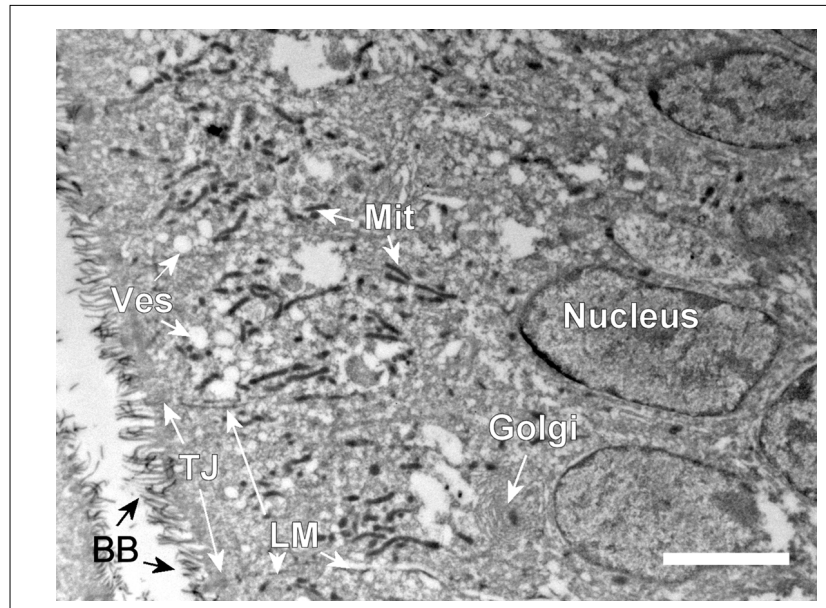

FIGURE 2 | Transmission electron micrograph from the middle intestine of SW-acclimated Atlantic salmon. The apical brush border is seen to the left (BB). Tight junctions (TJ), lateral membrane (LM), cytoplasmic vescicles (Ves), mitochondria (Mit), nucleus, and Golgi apparatus are indicated. Size bar $=5 \mu \mathrm{m}$.
(Figure 4A). All pharmacological agents significantly inhibited $J_{v}$ when tested in FW and/or SW intestines: ouabain (FW): 66\%; bumetanide (FW): 67\%; ouabain (SW): 72\%; bumetanide (SW): $82 \%$; KCN (SW): $68 \%$; phloridzin (SW): $20 \%$; TEA (SW): $20 \%$ (Figure 4).

In the middle intestine, mercury (Figure 5) inhibited $J_{v}$, when added mucosally (42\%) and when added to both sides simultaneously $(70 \%)$. In the posterior segment the effect of mercury was somehow different: there was a tendency that mucosal addition of mercury increased $J_{v}(p=0.062)$, had no effect when added serosally, whereas a strong inhibitory effect $(78 \%)$ was observed when added to both sides simultaneously. Mercaptoethanol completely abolished all observed effects of mercury except when added mucosally in the posterior segment, where it reinforced the mercury effect.

\section{IMMUNOHISTOCHEMISTRY AND CONFOCAL MICROSCOPY}

In pyloric caeca there were distinct immunoreactions to all three aquaporins (Aqplaa, Aqplab, and Aqp8ab) in the brush border membrane (Figure 6). Aqp laa and - $1 \mathrm{ab}$ also stained positive in the sub-apical domain of enterocytes, whereas Aqp8ab was restricted to the outermost zone with microvilli.

Aqplaa immunoreaction was confined to the apical brush border zone in the middle and posterior intestine (Figure 7). The intensity of the reaction was generally not very strong. Occasionally, goblet cells also stained positive for this aquaporin in the middle but not in the posterior segment or pyloric caeca.

Staining for Aqplab was also found in the brush border throughout the intestine (Figure 8). In the middle segment it was confined to the outermost zone with microvilli, with almost no sub-apical staining. The sub-apical cytoplasmic zone was

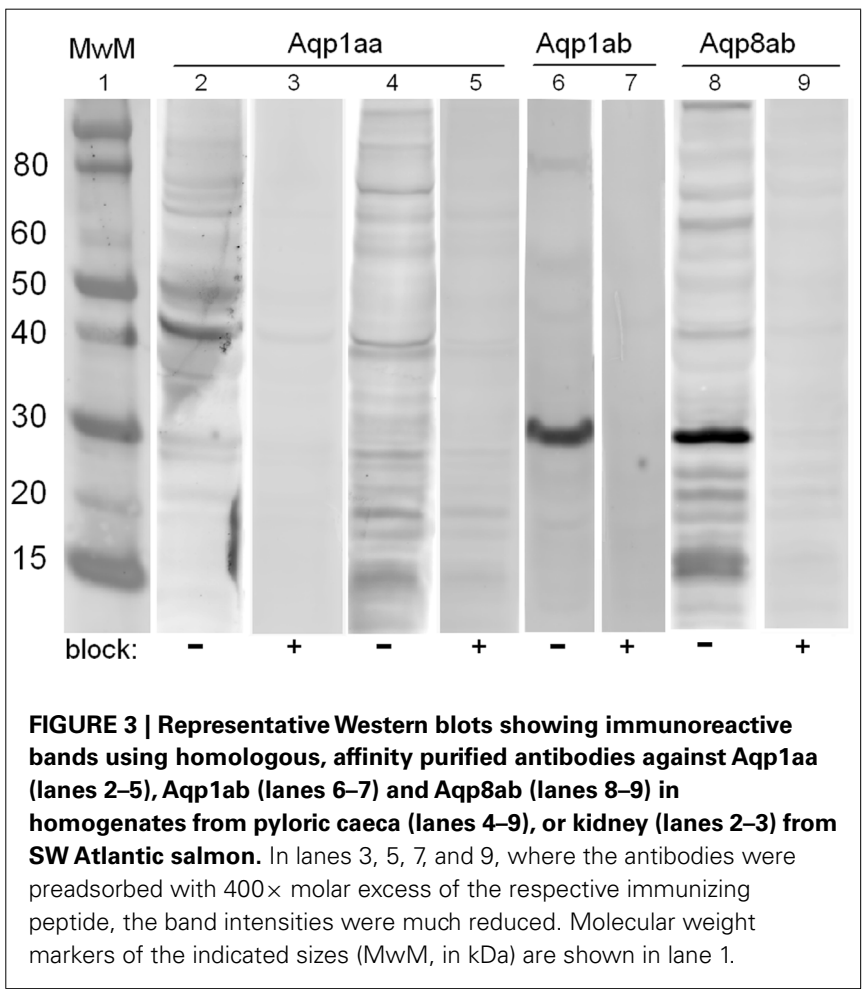



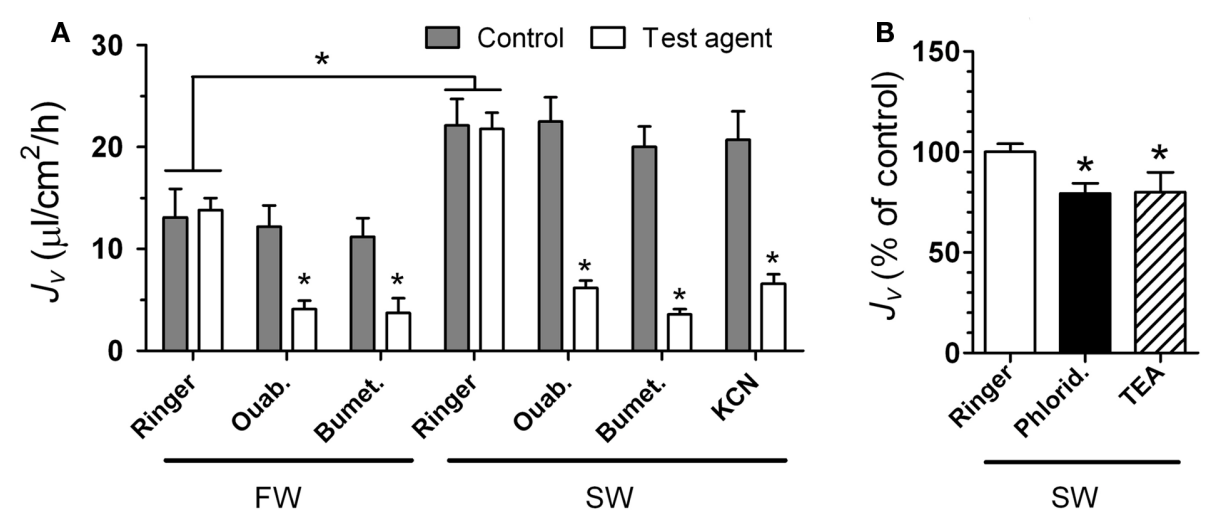

FIGURE 4 |The effect of various inhibitors on iso-osmotic water transport $\left(\boldsymbol{J}_{\boldsymbol{v}}\right)$ in the intestine of rainbow trout. In (A) trout were acclimated to either FW or SW and non-everted sacs of the posterior intestine were used for the measurements of $J_{v}$ (shown in $\mu \mathrm{L} \mathrm{cm}^{-2} \mathrm{~h}^{-1}$ ): ouabain (Ouab) was added to the serosal side; bumetanide (Bumet), and $\mathrm{KCN}$ were added to the mucosal side. Following the control measurement, intestinal sacs were incubated for $30 \mathrm{~min}$ with the test agent before the effect was evaluated. All inhibitors were applied at $10^{-4} \mathrm{M}$ concentrations. In (B) whole (i.e., middle + posterior) intestines from SW trout were used to measure $J_{v}$ (shown in \% of control): phloridzin $\left(1 \mathrm{mmol} \mathrm{L}^{-1}\right)$ or TEA ( $\left.10 \mathrm{mmol} \mathrm{L}^{-1}\right)$ were added to the mucosal side. Asterisk indicates difference from control value (ringer; $p<0.05$ ). All values represent mean \pm SEM, $n=6-8$. significantly stained, however, in the posterior intestine-especially in the most distal part near the rectum.

Aqp8ab gave very strong staining intensity of the intestinal brush border (Figure 9), and in the posterior segment staining of peri-nuclear and lateral membrane structures was also revealed especially when using higher magnification (Figure 10). In the lateral membrane Aqp8ab co-localized with the $\mathrm{Na}^{+}, \mathrm{K}^{+}$-ATPase. No such lateral staining was seen using the Aqplaa and - lab antibodies.

The aquaporin sequences were subject to analysis for potential phosphorylation sites by using the NetPhos 2.0 server (www.cbs.dtu.dk; Blom et al., 1999; Figure 11). Aqplaa has one tyrosine residue with high phosphorylation probability, whereas Aqplab has four serine residues with high probability near the cytoplasmic C-terminal. Aqp8ab has no phosphorylation sites in the cytoplasmic C-tail; however, a single threonine residue near the $\mathrm{N}$-terminus and a single serine residue in the second cytoplasmic loop have high phosphorylation potential.

\section{DISCUSSION}

In the intestine of marine fish, osmotic water absorption across the luminal epithelium may occur via one or more of different routes: paracellularly between enterocytes or transcellularly across the lipid bilayers, through aquaporins or as co-transport with ions and nutrients (Laforenza et al., 2005; see Figure 12). The present study focused on a pharmacological characterization of this transport mechanism and suggests the contribution of three Aqp paralogs in the salmon intestine: Aqp1aa, -1ab, and -8ab. Among these the role of Aqp8ab is particularly interesting, since it has not been demonstrated before. It is strongly increased in response to SW-transfer (Tipsmark et al., 2010) and it is expressed both apically and laterally in enterocytes.

\section{PHARMACOLOGICAL CHARACTERIZATION OF WATER TRANSPORT}

In a series of in vitro experiments, we characterized the nature of iso-osmotic water transport across the intestinal epithelium in rainbow trout. The reason for using rainbow trout was that they could be obtained in a size that was reasonable for studies of this kind. Furthermore, there is no reason to speculate different mechanisms for water absorption in different salmonids. Isoosmotic solutions were used on both sides of the intestine, thus any observed net water transport must occur as a result of osmotic gradients established on a micro scale. SW-fish generally had higher $J_{v}$ than FW fish, indicating that the intestinal epithelium is modified in response to hyper-osmotic exposure. This is in accordance with earlier studies in salmonids (Collie and Bern, 1982; Veillette et al., 1993) and is also seen during smoltification in preparation to seaward migration (Sundell et al., 2003). As expected, $J_{v}$ was significantly inhibited by serosal addition of ouabain and mucosal addition of bumetanide indicating the involvement of both basolateral $\mathrm{Na}^{+}, \mathrm{K}^{+}$-ATPase and apical absorptive $\mathrm{Na}^{+}, \mathrm{K}^{+}$, $2 \mathrm{Cl}^{-}$-co-transport in the process. Furthermore, the metabolic poison $\mathrm{KCN}$ also inhibited $J_{v}$. None of the agents inhibited $J_{v}$ by $100 \%$ within the timescale used here but the findings are in accordance with the standing gradient model for transepithelial water transport (see Larsen and Møbjerg, 2006). This mechanism has also been suggested, although not directly proven for coho salmon, $O$. kisutch, and Atlantic salmon, where ouabain was shown to decrease the $J_{v}$ across intestinal sac preparations by $67-100 \%$ (Collie and Bern, 1982; Veillette et al., 1993).

Our experiments with mercury chloride revealed that water transport was significantly inhibited by this compound, both when added serosally and mucosally. A puzzling exception to this was seen in the posterior segment, where mercury did not have a significant effect when added only mucosally. The highest degree of inhibition was, however, obtained when added to both sides simultaneously in both middle and posterior segments. This effect was reversibly blocked by mercaptoethanol. Based on osmotic swelling assays with heterologously expressed aquaporins in Xenopus oocytes, mercury has been shown to inhibit water transport by certain Aquaporin orthologs (AQP1 in mammals: e.g., Savage and Stroud, 2007; Aqp1, Aqp1dup, Aqp3, Aqpe in European eel: 

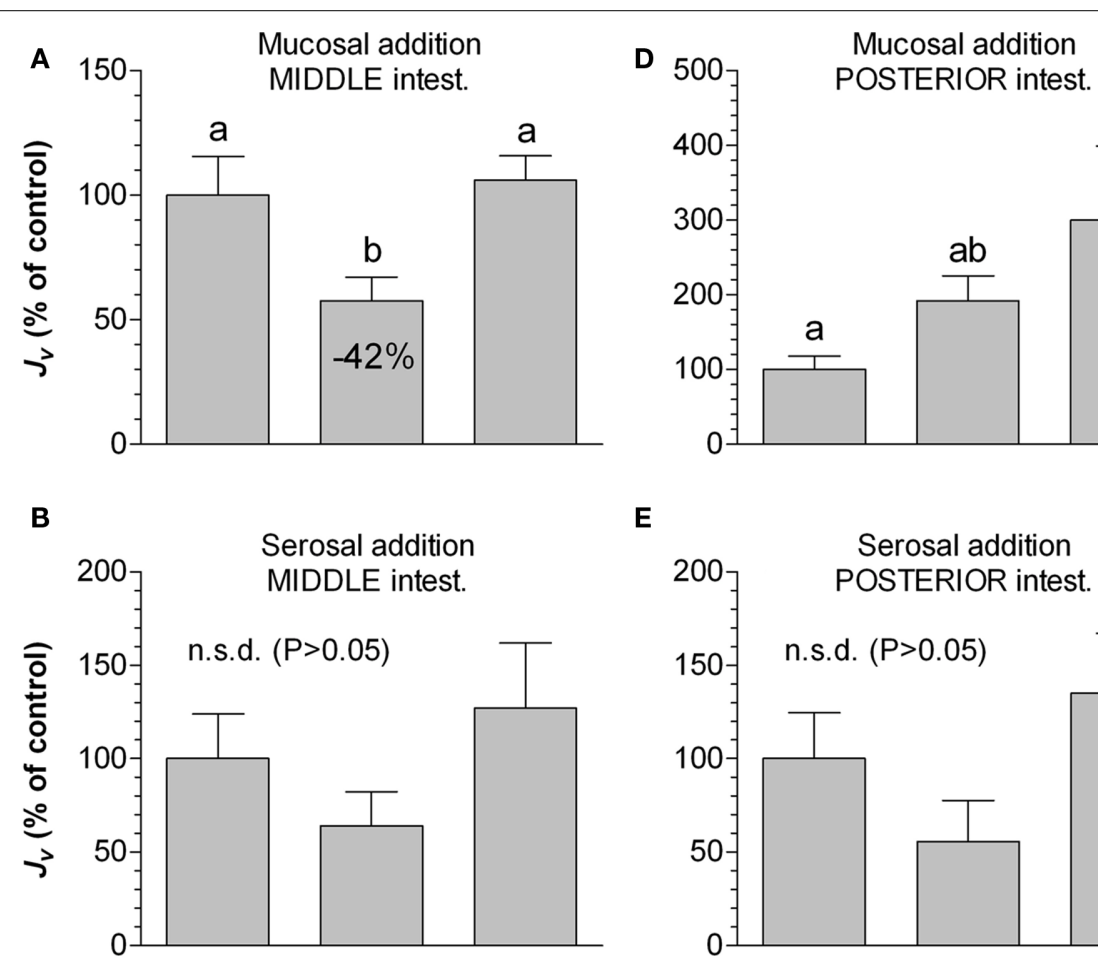

E
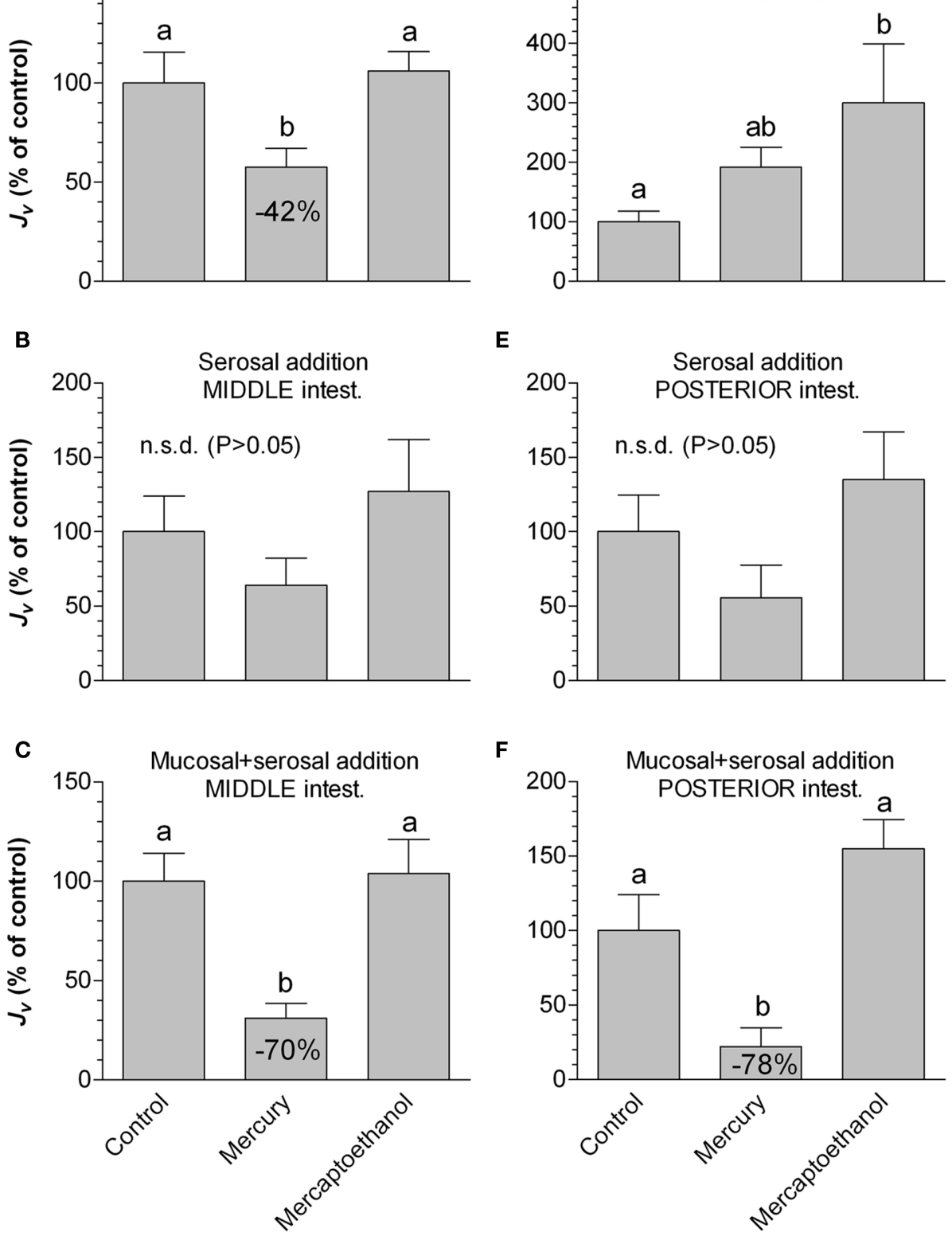

FIGURE 5 |The effect of mercury and subsequent mercaptoethanol on water transport rate $\left(J_{v}\right.$ in $\%$ of control values) in non-everted sacs of middle (A-C) and posterior (D-F) segments of intestine from SW-acclimated rainbow trout. In different series the test agents were added to either the mucosal (A-D), serosal (B-E), or both sides simultaneously (C-F). Bars sharing identical letters are not significantly different ( $p>0.05)$. Values represent mean \pm SEM, $n=8$.

MacIver et al., 2009) by interacting with the central pore (Savage and Stroud, 2007). In the eel study, the degree of inhibition by mercury was $54-64 \%$ for each individual aquaporin. It has also been reported that mercury inhibits water transport through AQP8 100\% when reconstituted into proteoliposomes (Liu et al., 2006). Therefore, our findings support the involvement of aquaporins in water transport in the salmonid intestine. However, data based on studies in intact epithelia should be interpreted with great care, as mercury is likely to interact with other important elements of water transport such as $\mathrm{Na}^{+}, \mathrm{K}^{+}$-ATPase (Wang and Horrisberger, 1996), $\mathrm{Na}^{+}, \mathrm{K}^{+}, 2 \mathrm{Cl}^{-}$-co-transporter (Kinne-Saffran and Kinne, 2001) and also the status of tight junction proteins (Kawedia et al., 2008). Therefore alternative aquaporin inhibitors should be tested as well. In some studies it has been reported that quaternary ammonium compounds such as TEA inhibit water transport through AQP1, -2 and -4 but not AQP3 and -5 when expressed in Xenopus oocytes (AQP1: Brooks et al., 2000; Detmers et al., 2006) and mammalian cells (AQP1: Yool et al., 2002). We found 

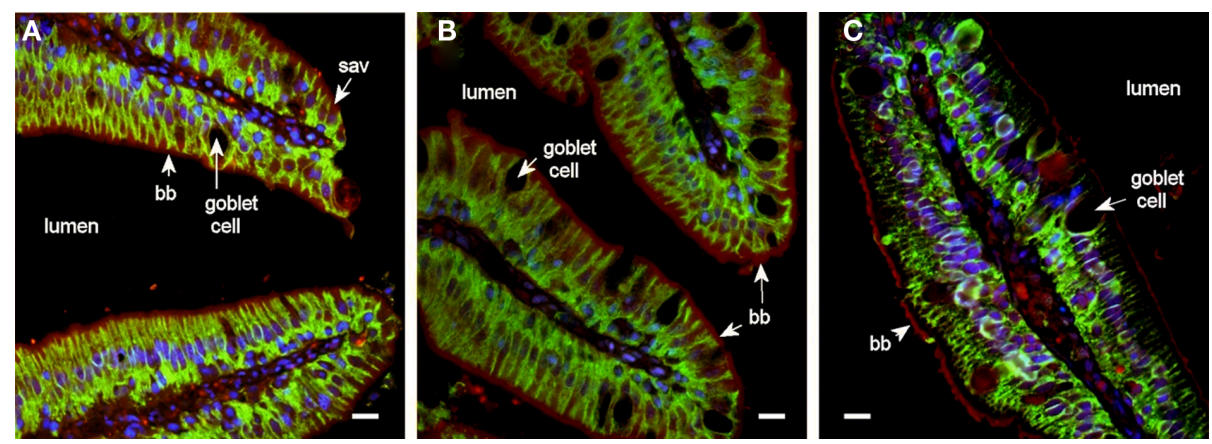

FIGURE 6 | Fluorescent microscope images (40x objective) showing localization of Aqp1aa (A), Aqp1ab (B), and Aqp8ab (C) in pyloric caeca from SW-acclimated Atlantic salmon. Sections were incubated with antibodies against the aquaporin (red), $\mathrm{Na}^{+}, \mathrm{K}^{+}$-ATPase (green), and nuclei were counterstained with DAPI (blue). Lumen, goblet cells, nuclei, brush border (bb), and sub-apical vesicles (sav) are indicated. Note the distinct immunoreaction to all three aquaporins in the brush border and for Aqp1aa and $-1 \mathrm{ab}$ also in the sub-apical cytoplasmic domain. Size bar $=20 \mu \mathrm{m}$.
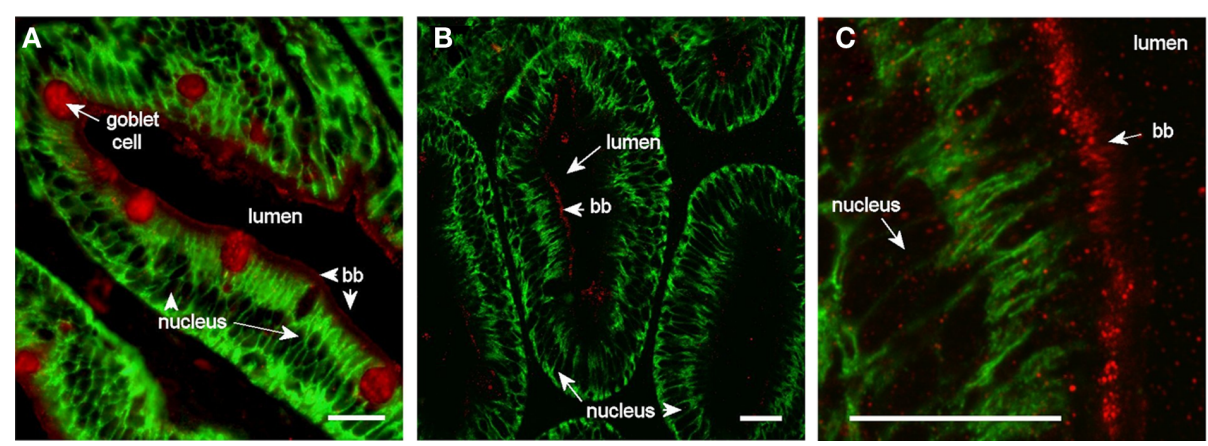

FIGURE 7 | Confocal images (63x objective) showing localization of Aqp1aa in middle (A) and posterior $(B, C)$ segments of intestine from SW-acclimated Atlantic salmon. Sections were incubated with a cocktail of antibodies against Aqp1aa (red) and $\mathrm{Na}^{+}, \mathrm{K}^{+}$-ATPase (green). Lumen, goblet cells, nuclei, and brush border (bb) are indicated. Size $\mathrm{bar}=20 \mu \mathrm{m}$.
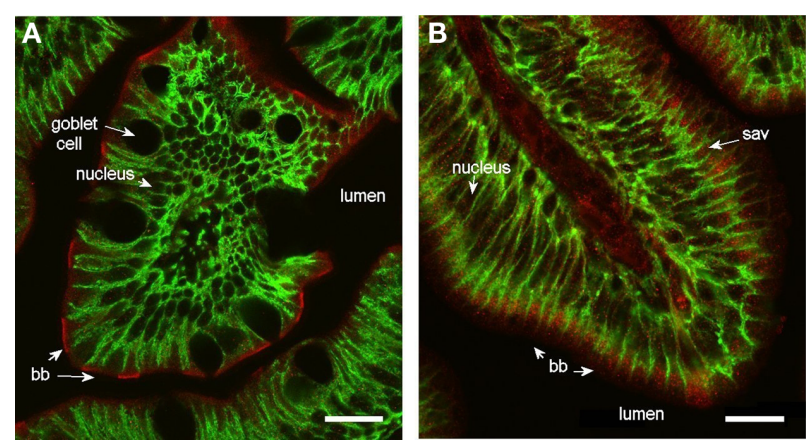

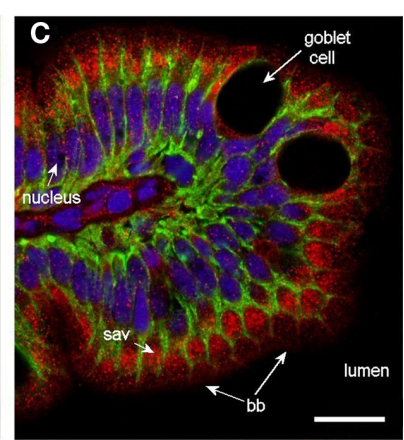

FIGURE 8 | Confocal images (63x objective) showing localization of Aqp1ab in middle (A) and posterior segments $(B, C)$ of intestine from SW-acclimated Atlantic salmon. $(B, C)$ are from the proximal and distal region of the posterior segment, respectively, with reference to the ileo-rectal valve. Sections were incubated with a cocktail of antibodies against Aqp1ab (red), $\mathrm{Na}^{+}, \mathrm{K}^{+}$-ATPase (green), and nuclei were counterstained with DAPI [blue, only in (C)]. Lumen, goblet cells, nuclei, brush border (bb), and sub-apical vesicles (sav) are indicated. Size bar $=20 \mu \mathrm{m}$. a $14 \%$ inhibition of $J_{v}$ by $10 \mathrm{mmol} \mathrm{L}^{-1}$ TEA, thus adding to the evidence that aquaporins are involved in water transport. A possible explanation for the relatively small inhibition by TEA is the fact that several aquaporins are expressed in the intestine, some of which may not be sensitive to TEA. TEA also inhibits potassium channels even though the potency is 1000 -fold lower (Detmers et al., 2006).

We also tested the effect of phloridzin which is a recognized inhibitor of the apical sodium-glucose symporter (SGLT1; Xia et al., 2003). The effect was marginal when compared to the effects 

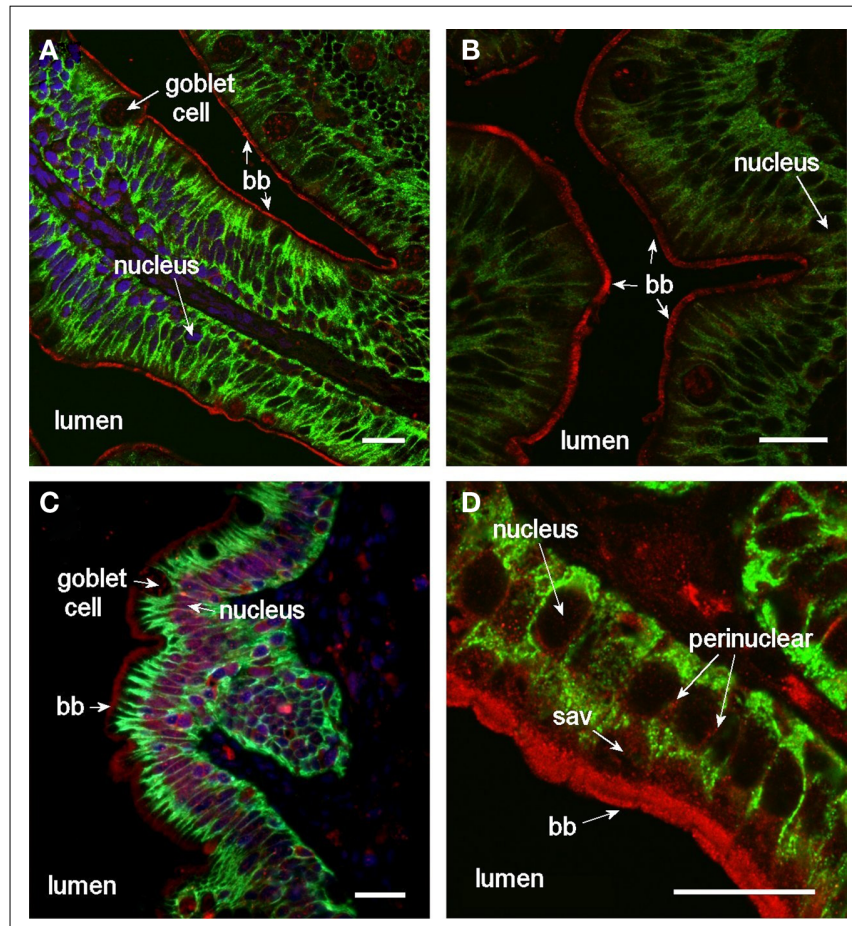

FIGURE 9 | Confocal images (63x objective) showing localization of Aqp8ab in middle $(A, B)$ and posterior $(C, D)$ segments of intestine from SW-acclimated Atlantic salmon. Sections were incubated with a cocktail of antibodies against Aqp8ab (red), $\mathrm{Na}^{+}, \mathrm{K}^{+}$-ATPase (green), and nuclei were counterstained with DAPI [blue, in (A-C)]. Lumen, goblet cells, nuclei, brush border, (bb) and sub-apical vesicles (sav) are indicated. Size bar $=20 \mu \mathrm{m}$.

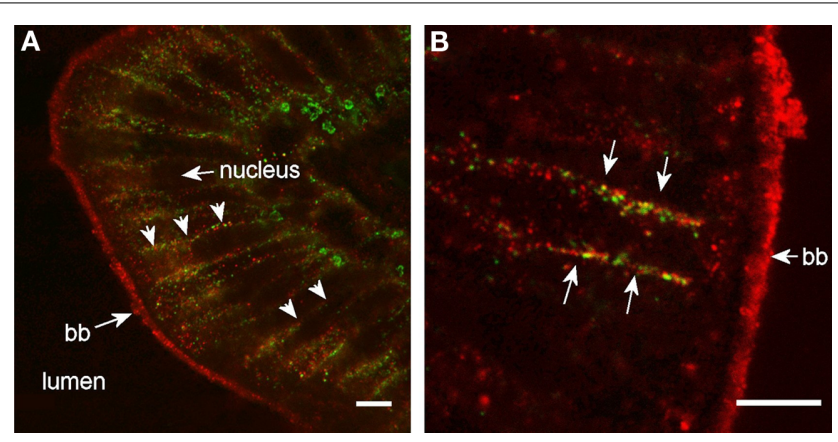

FIGURE 10 | High magnification confocal images (100x objective) showing localization of Aqp8ab in the posterior segment of intestine from SW-acclimated Atlantic salmon. Sections were incubated with a cocktail of antibodies against Aqp8ab (red) and $\mathrm{Na}^{+}, \mathrm{K}^{+}$-ATPase (green). Lumen, nuclei, brush border (bb), and lateral membranes (arrows) are indicated. Size bar $=20 \mu \mathrm{m}$.

of all other agents, indicating that water transport accompanying the function of this protein is not a major route. We tested the effect on both fed and starved fish but found no difference (Madsen, unpublished data). We suspect that this transporter may have a more significant role in water transport in the anterior part of the intestine (including pyloric caeca), where nutrient absorption is assumed to be higher. Unpublished observations in our laboratory have shown that the SGLT1 transcript is expressed in all intestinal segments - the order of expression being pyloric caeca $>$ middle $>$ posterior intestine. This supports a previous observation of the presence of the SGLT1 protein in the brush border of absorptive enterocytes in the rainbow trout (Polakof et al., 2010).

\section{VALIDATION OF AFFINITY PURIFIED ANTIBODIES}

Western blotting experiments on homogenates from intestinal tissues revealed immunoreactive bands around $30 \mathrm{kDa}$ for all three aquaporins: Aqp1aa, Aqplab, and Aqp8ab in addition to both lower (15 kDa: Aqp8ab) and higher (40 kDa: Aqp1aa) molecular weight bands. The bands around 30 were efficiently blocked when the antibodies were preadsorbed with $400 \times$ molar excess of the specific antigenic peptide, suggesting that the affinity purified antibodies recognize specific aquaporin isoforms with the expected $\mathrm{Mr}$ around $30 \mathrm{kDa}$. However, some degree of blocking was also observed for both higher and lower Mr bands. We cannot presently explain the bands at $15-20 \mathrm{kDa}$, whereas it is well known that aquaporins appear in various glycosylated forms that may give rise to multiple bands $>30 \mathrm{kDa}$ on Western blots (e.g., Christensen et al., 2000; Pandey et al., 2010). Aquaporins are also known to be functionally arranged in tetramers in biological membranes; however, there was no indication of oligomeric forms when using the present reducing conditions. The Aqplab and -8ab antibodies seemed to have a better quality for Western blotting than the Aqplaa antibody, which also gave the weakest signals. The data for Aqplaa thus have to be interpreted with some care.

\section{LOCALIZATION OF INTESTINAL AQUAPORINS: Aqp1}

Several studies have shown that expression of Aqp1 is higher in SW-acclimated fish compared to FW fish (Aoki et al., 2003; Martinez et al., 2005b; Raldúa et al., 2008; Kim et al., 2010), suggesting that it is involved in the absorption of imbibed water in these species. This is also the case in Atlantic salmon judged from higher mRNA levels in SW-fish than FW fish (Tipsmark et al., 2010). The present study used SW salmon for the localization of aquaporins. Aqplaa was localized in the apical brush border membranes of enterocytes along the whole length of the intestine. Even though fluorescence staining intensities must be compared with care, it generally appeared that the staining intensity for Aqplaa was weaker than for the other aquaporins investigated. This matches the weaker bands observed on the Western blots when probing for this aquaporin and we cannot exclude that it is due to a poorer antibody quality (see above). In the pyloric caeca, staining was also observed in the sub-apical zone of enterocytes, suggesting the presence of cytoplasmic vesicles with an internalized pool of Aqplaa. This was less prominent in the other regions. In the middle intestine a significant immunoreaction was also observed in goblet cells, which has not been shown in previous studies. Interestingly, this was not seen in other segments of the intestine even though goblet cells were numerous there. Nor did the antibodies for Aqp lab and Aqp8ab produce a similar reaction. Localization of Aqp3 in goblet cells has been reported in the posterior esophagus and the rectum of European eel (Cutler et al., 2007), suggesting that aquaporins may to some degree be involved in mucus production or maintenance of the mucus layer fluidity. 


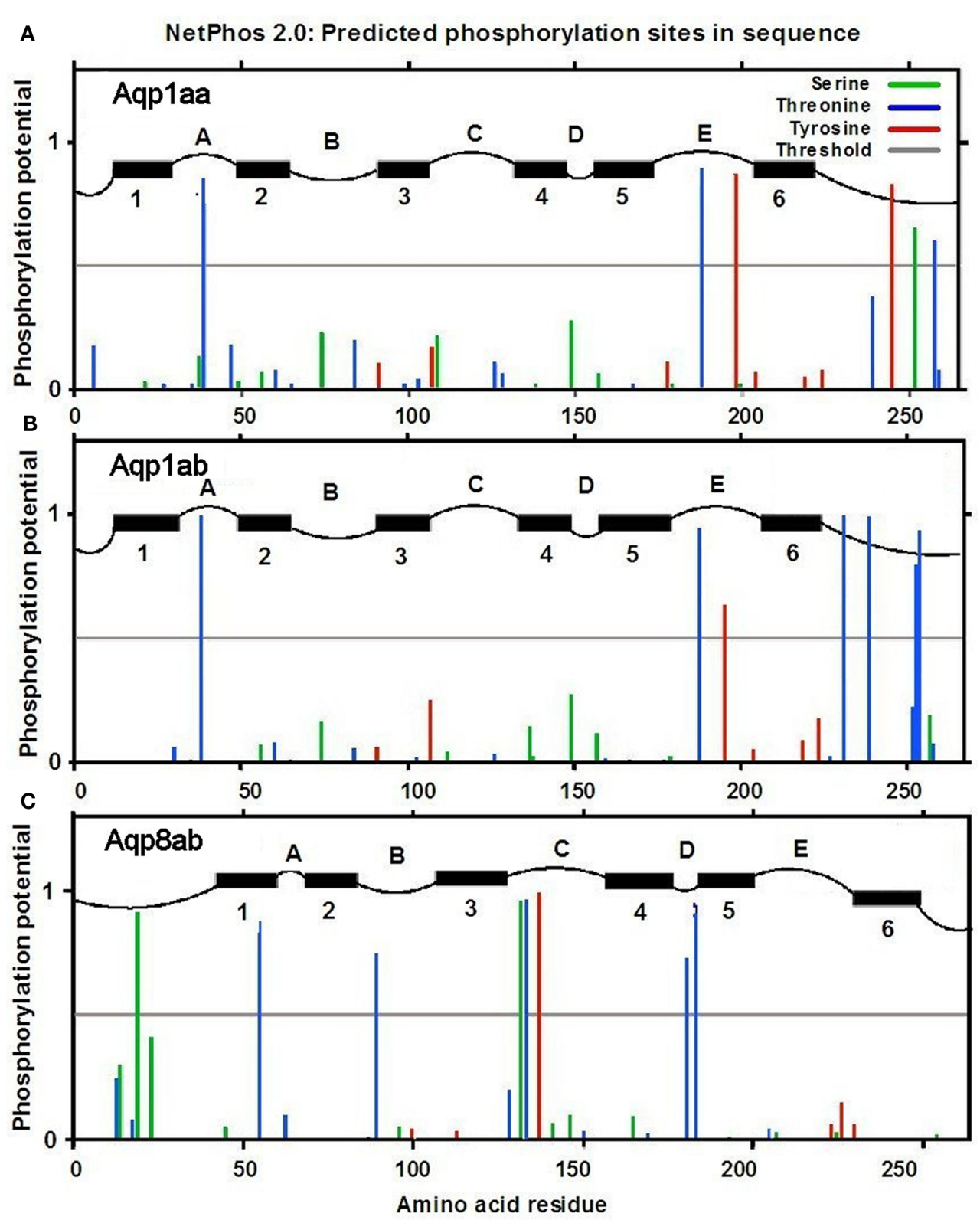

FIGURE 11 | Analysis of predicted serine, threonine and tyrosine phosphorylation sites in Aqp1aa (A), Aqp1ab (B), and Aqp8ab (C) (from NetPhos 2.0 server at www.cbs.dtu.dk; Blom et al., 1999). Black horizontal bars indicate predicted transmembrane domains with numbering below. Letters (A-E) indicate extracellular domains; letters $(B-D)$ indicate cytosolic domains (from TMPred at www.ch.embnet.org; Hofmann and Stoffel, 1993).
Aqplab was also present in the apical brush border in all segments of the intestine, with a very distinct staining of the outermost zone of microvilli in the pyloric caeca and middle intestine. In the posterior intestine the localization moved into a more sub-apical position, and was most pronounced in the distal region of the posterior intestine, where Aqplab was located almost exclusively in sub-apical compartments. Based on the transmission electron microscopic picture (Figure 2) this region was judged to contain intracellular vesicles which may form a pool of recruitable membrane proteins including aquaporins.
Other fish studies have also localized Aqp1 in the enterocytes of the intestinal epithelium: Aqplaa was demonstrated in the apical membrane of the enterocytes in Japanese and European eel (Aoki et al., 2003; Martinez et al., 2005a). In gilthead seabream, Raldúa et al. (2008) investigated the localization of both Aqplaa and $1 \mathrm{ab}$, and found immunoreactivity of both paralogs in the apical brush borders in the duodenum and hindgut, with Aqplaa being most abundant. This aquaporin may thus facilitate water entry into enterocytes. Aqp laa also showed weak staining of the lateral membranes and the peri-nuclear region in their study, suggesting that this paralog may also offer an exit pathway for absorbed water in 


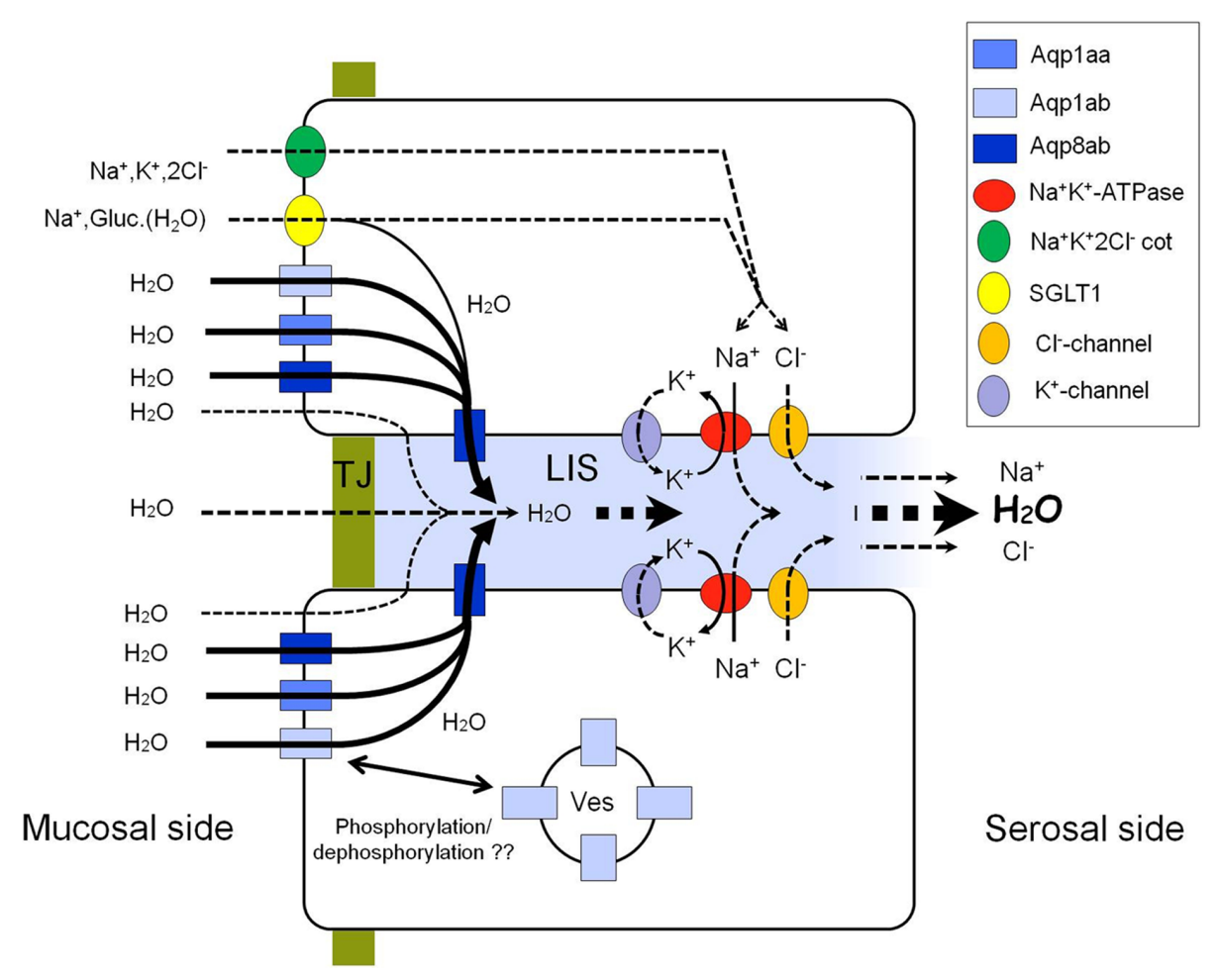

FIGURE 12 | Tentative model of the pathways of water absorption across the intestinal epithelium in salmonids. An osmotic gradient is set up across the lateral membrane by means of the $\mathrm{Na}^{+}, \mathrm{K}^{+}$-ATPase. Water may be drawn osmotically through four pathways: (1) diffusion through the lipid bilayer of the enterocyte; (2) paracellular diffusion through the TJ; (3) symport with $\mathrm{Na}^{+}$and glucose by the SGLT1; (4) diffusion through aquaporins apically as well as laterally. Aquaporins may be translocated between sub-apical vesicles and the cell membrane possibly regulated by phosphorylation events. The model is based on the elements investigated in the present paper. For simplicity apical ion-transporters are only shown in the upper enterocyte. LIS, lateral intercellular space; TJ, tight junction; Ves, intracellular vesicles. the seabream. In the seabream rectum (equals the posterior intestine investigated in the present study) Aqplaa was preferentially localized in sub-apical intracellular compartments of enterocytes. Aqplab on the other hand stained the apical brush borders in the rectal epithelium intensively (Raldúa et al., 2008).

The results of the present study are thus overall in agreement with previous fish studies with respect to localization of Aqp1 paralogs in enterocytes. However, the relative staining intensities between segments seem to vary between species (seabream: Raldúa et al., 2008; European eel: Martinez et al., 2005a). The presently observed change of Aqplab localization when moving from the middle into the posterior segment, and especially within the posterior segment shows, that the definition of intestinal "segments" is purely based on gross morphology and does not necessarily imply similar function or cellular histology within a given segment. Taken together, all available data show that Aqp1 paralogs may be important for trans-enterocytic water transport, and in most species offers an entry pathway through the apical membranes.

In the GIT of mammals, AQP1 is typically localized in the serosa, in endothelia of the lymphatic vessels and in the lamina propria but not in the epithelium lining the intestinal lumen (rats: Koyama et al., 1999; Ma and Verkman, 1999). On the other hand studies on dolphins (Suzuki, 2010) and the desert rodent Octodon degus (Gallardo et al., 2002) have localized AQP1 in the apical membranes of the epithelial cells lining the gut, similar to what is seen in marine fish. Thus this aquaporin may be of greater importance for water uptake in the GIT of animals living in more water-deprived environments.

\section{Aqp8}

The transport characteristics of mammalian AQP8 is different from the orthodox aquaporins as it is able to transport ammonia (Liu et al., 2006; Carbrey and Agre, 2009) and urea (Ma et al., 1997; Cerdà and Finn, 2010) in addition to water. Our study demonstrated that the Aqp8ab protein is abundant in the brush border membrane of enterocytes in all three intestinal segments of Atlantic salmon. The Aqp8ab antibody gave a very strong band around $30 \mathrm{kDa}$ and seemed the most abundant of the three aquaporins investigated. This observation strongly supports a physiological role of Aqp8ab in water absorption across the apical membrane of the intestinal epithelia in SW salmon. Three other studies have reported aqp 8 mRNA expression in fish. Kim et al. (2010) recently reported expression of an aqp8 paralog in the intestine of Japanese eel, which increased after SWacclimation. Tingaud-Sequeira et al. (2010) found expression of two paralogs (aqp8aa and aqp8ab) in the intestine and one para$\log (a q p 8 b)$ in the brain of zebrafish. And finally Tipsmark et al. (2010) reported two paralogs of aqp8 in Atlantic salmon: aqp8aa, 
which is exclusively expressed in the liver and aqp8ab (formerly named AQP8b), which is expressed exclusively in the intestine (pyloric caeca, middle and posterior intestine). At present, two observations suggest that Aqp8ab is important for intestinal water transport in Atlantic salmon: (1) aqp $8 a b$ mRNA is up-regulated during SW-acclimation in pyloric caeca, middle, and posterior intestine, and (2) aqp8ab mRNA expression is increased during smoltification in preparation for SW-entry (Tipsmark et al., 2010). From the present study, it is further evident that Aqp8ab co-localizes with $\mathrm{Na}^{+}, \mathrm{K}^{+}$-ATPase in the lateral membranes in the middle and posterior intestine, thus Aqp8ab may be important for both water entry and exit through the enterocyte. We also found occasional peri-nuclear staining using the Aqp8ab antibody, which may be related to recognition of Aqp8ab protein in association with the Golgi apparatus and endoplasmic reticulum. Investigations using colloidal gold staining and transmission electron microscopy are currently performed in order to verify this lateral staining.

Mammalian studies have detected AQP8 protein expression in liver, testis, epididymis, kidney, bronchi/trachea, duodenum, jejunum, and colon (Elkjær et al., 2001). AQP8 is found to be important in the secretion of bile from liver hepatocytes, where it is recruited from intracellular compartments to the plasma membrane following stimulation with glucagon (Gradilone et al., 2003) or cAMP (Carcia et al., 2001). The molecular mechanism behind this trafficking is not yet fully understood, even though there is evidence that phosphorylation of AQP8 initiates its translocation from the intracellular compartment to the plasma membrane (Carbrey and Agre, 2009). A study by Elkjær et al. (2001) and Calamita et al. (2001) localized AQP8 in the intestinal epithelium; however, it was located almost exclusively in intracellular compartments. Later studies on rats by Tritto et al. (2007) and Laforenza et al. (2005) also revealed the expression of AQP8 in the small intestine and colon, respectively. In these studies AQP8 was localized in the brush borders of the epithelial cells. All these studies suggest that AQP8 may play a role in the movement of water across the apical membranes of the intestinal cells, and that trafficking of AQP8 between intracellular compartments and the plasma membrane might be involved in the regulation of this aquaporin.

\section{ANALYSIS OF POTENTIAL PHOSPHORYLATION SITES}

In the evolutionary history of teleosts, it is suggested that the group arose in FW and later inhabited the marine environment (Fyhn et al., 1999). This event required new molecular adaptations to maintain water homeostasis. Among such adaptations, gene duplication of $a q p 1$ at a local gene level rather than at the chromosome level has been suggested (Tingaud-Sequeira et al., 2008, 2010; Finn and Cerdà, 2011). One mechanism that is known to regulate trafficking of aquaporins is phosphorylation events, exemplified in the scenario of fish oocyte hydration. In both the marine gilthead seabream (Fabra et al., 2005, 2006; TingaudSequeira et al., 2008) and the FW catfish (Heteropneustes fossilis, Chaube et al., 2011) the Aqplab paralog is expressed in oocytes, where it has a particular role to facilitate oocyte swelling during meiotic maturation. In seabream, this is initiated when Aqp lab is recruited to the oocyte cell surface induced by de-phosphorylation of its C-terminal serine-254, whereas in catfish phosphorylation of serine-227 induces membrane insertion of this paralog. Interestingly, in seabream, only the Aqp 1 ab paralog has a phosphorylation site, Aqplaa does not. In salmon, both Aqplaa and - lab have predicted phosphorylation sites in the cytosolic domain between residues 230 and 260 (Figure 11), however, the phosphorylation potential is stronger for Aqplab than Aqplaa at especially serine residues. We suspect that some of these sites may be involved in regulatory phosphorylation, which may affect the cellular distribution of these aquaporins. Judged from the transmission electron microscopy picture (Figure 2) vesicles are abundant in the subapical zone of the enterocyte. This is also where especially Aqp 1ab is located in the enterocytes of the distal part of the posterior intestine, suggesting that trafficking may be an important aspect of the function of this aquaporin in this region. Sup-apical localization of Aqplaa was not seen to the same degree. Aqp8ab was also localized in sub-apical domains in the present study (Figure 9D) suggesting a vesicular location. However, no phosphorylation residues were present near the C-terminus of this aquaporin; only a serine, a threonine and a tyrosine residue with a high phosphorylation potential were present in intracellular domains of Aqp8ab, and it thus differs markedly from the aquaporin $1 \mathrm{aa}$ and $-1 \mathrm{ab}$ in this respect. It shall be interesting to investigate the role of these residues in intracellular trafficking of Aqp8ab.

\section{CONCLUSION AND PERSPECTIVES: PHYSIOLOGICAL ROLE OF AQUAPORINS IN INTESTINAL WATER ABSORPTION}

There is increasing circumstantial evidence of a role of aquaporins in intestinal water uptake in SW-fish. The definite proof is, however, still missing - not least due to the pharmacology of aquaporins and the difficulty of specifically inhibiting these in live tissue. In salmon, the significant up regulation of aqp 1aa and especially - $8 a b$ mRNA in pyloric caeca, middle and posterior intestine, suggests their importance in intestinal water absorption in hypoosmoregulating fish (Tipsmark et al., 2010). In addition, there may be a small contribution to apical water transport through supplementary pathways such as the SGLT1, though depending on the nutritional condition. This study has shown that Aqplaa, -1ab, and $-8 \mathrm{ab}$ are localized in the apical brush borders of enterocytes lining the entire intestine from the pyloric caeca through the anus, thus allowing entry of water from the lumen into the enterocytes. In salmon, the pyloric caeca may have a particular significance in quantitative uptake of water (and nutrients; Veillette et al., 2005). We could not confirm a lateral location of Aqp1 as reported in seabream (Raldúa et al., 2008) but give evidence that in salmon (and rainbow trout: Madsen, unpublished data) Aqp8ab occupies this location - in addition to being present in both apical and sub-apical locations. Thus at least for salmonids it is possible to propose a model for transcellular water transport involving three aquaporin paralogs (Figure 12). The reason for having (at least) three different paralogs accounting for apical water entry can only be speculated but such diversity is also common in mammalian tissues. One obvious possibility is that paralogs may have different permeability characteristics and regulatory potential and thus contribute differently to long- and short-term regulation. We are currently investigating the permeability characteristics of the expressed isoforms by heterologous expression in Xenopus oocytes. 
During the progress of this work we have identified yet another Aqp8 paralog - termed Aqp8b in the extensive EST library of Atlantic salmon. This leads to three paralogs of Aqp8, which is also the case for zebrafish (Tingaud-Sequeira et al., 2010). The Aqp8b isoform is highly expressed in the GI tract and kidney (Madsen, unpublished data) and future studies will reveal the localization and contribution of this novel paralog to intestinal water transport.

\section{REFERENCES}

Aoki, M., Kaneko, F., Hasegawa, S., Tsutsui, N., and Aida, K. (2003). Intestinal water absorption through aquaporin 1 expressed in the apical membrane of mucosal epithelial cells in seawater-adapted Japanese eel. J. Exp. Biol. 206, 3495-3505.

Blom, N., Gammeltoft, S., and Brunak, S. (1999). Sequence- and structurebased prediction of eukaryotic protein phosphorylation sites. J. Mol. Biol. 294, 1351-1362.

Brooks, H. L., Regan, J. W., and Yool, A. J. (2000). Inhibition of aquaporin-1 water permeability by tetraethylammonium: involvement of the loop E pore region. Mol. Pharmacol. 57, 1021-1026.

Calamita, G., Mazzone, A., Bizzoca, A., Cavalier, A., Cassano, G., Thomas, D., and Svelto, M. (2001). Expression and immunolocalization of the aquaporin-8 water channel in rat gastrointestinal tract. Eur. J. Cell Biol. 80, 711-719.

Carbrey, J. M., and Agre, P. (2009). "Discovery of the aquaporins and development of the field," in Aquaporins (Handbook of Experimental Pharmacology) 190, ed. E. Beitz (Berlin: Springer-Verlag), 3-28.

Carcia, F., Kierbel, A., Larocca, M. C., Gradilone, S. A., Splinter, P., LaRusso, N. F., and Marinelli, R. A. (2001). The water channel aquaporin-8 is mainly intracellular in rat hepatocytes, and its plasma membrane insertion is stimulated by cyclic AMP. J. Biol. Chem. 276, 12147-12152.

Cerdà, J., and Finn, R. N. (2010). Piscine aquaporins: an overview of recent advances. J. Exp. Zool. 313A, 1-28.

Chaube, R., Chauvigné, F., TingaudSequeira, A., Joy, K. P., Acharjee, A., Singh, V., and Cerdà, J. (2011). Molecular and functional characterization of catfish (Heteropneustes fossilis) aquaporin-1b: changes in expression during ovarian development and hormoneinduced follicular maturation. Gen. Comp. Endocrinol. 170, 162-171.

Christensen, B. M., Zelenina, M., Aperia, A., and Nielsen, S. (2000).
Localization and regulation of PKAphosphorylated AQP2 in response ito V-2-receptor agonist/antagonist treatment. Am. J. Physiol. Renal Physiol. 278, F29-F42.

Collie, N. L., and Bern, H. A. (1982). Changes in intestinal fluid transport associated with smoltification and sea-water adaptation in coho salmon, Oncorhynchus kisutch (Walbaum). J. Fish Biol. 21, 337-348.

Cutler, C. P., Martinez, A. S., and Cramb, G. (2007). The role of aquaporin 3 in teleost fish. Comp. Biochem. Physiol. 148A, 82-91.

Detmers, F. J., De Groot, B. L., Muller, E. M., Hinton, A., Konings, I. B., Sze, M., Flitsch, S. L., Grubmuller, H., and Deen, P. M. (2006). Quaternary ammonium compounds as water channel blockers - specificity, potency, and site of action. J. Biol. Chem. 281, 14207-14214.

Diamond, J. M., and Bossert, W. H. (1967). Standing-gradient osmotic water and solute transport in epithelia. J. Gen. Physiol. 50, 2061-2083.

Elkjær, M. L., Nejsum, L. N., Grecz, V., Kwon, T. H., Jensen, U. B., Frøkiær, J., and Nielsen, S. (2001). Immunolocalization of aquaporin- 8 in rat kidney, gastrointestinal tract, testis and airways. Am. J. Physiol. Renal Physiol. 281, F1047-F1057.

Fabra, M., Raldúa, D., Bozzo, M. G., Deen, P. M., Lubzens, E., and Cerdà, J. (2006). Yolk proteolysis and aquaporin-1o play essential roles to regulate fish oocyte hydration during meiosis resumption. Dev. Biol. 295, 250-262.

Fabra, M., Raldúa, D., Power, D. M., Deen, P. M., and Cerdà, J. (2005). Marine fish egg hydration is aquaporin-mediated. Science 307, 545.

Finn, R. N., and Cerdà, J. (2011). Aquaporin evolution in fishes. Front. Physiol. 2:44. doi: 10.3389/fphys.2011.00044

Fyhn, H. J., Finn, R. N., Reith, M., and Norberg, B. (1999). Yolk protein hydrolysis and oocyte free amino tive evolution of teleost fishes to seawater. Sarsia 84, 451-456. flow - a mechanism for coupling of acids as key features in the adap-

\section{ACKNOWLEDGMENTS}

Dr. B. M. Christensen and Ms. E.-M. Løcke (Department of Anatomy, Aarhus University, Denmark) are thanked for preparing the transmission electron micrograph in Figure 2. Drs J. R. Brewer and E. A. Christensen (Department of Biochemistry and Molecular Biology, SDU, Denmark) are thanked for assistance with the confocal microscopy. The project was supported by a grant from the Danish Natural Research Foundation (09-070689).

Gallardo, P., Olea, N., and Sepulveda, V. (2002). Distribution of aquaporins in the colon of Octodon degus, a South American desert rodent. Am. J. Physiol. Regul. Integr. Comp. Physiol. 283, R779-R788.

Giffard-Mena, I., Boulo, V., Aujoulat, F., Fowden, H., Castille, R., Charmantier, G., and Cramb, G. (2007). Aquaporin molecular characterization in the sea-bass (Dicentrarchus labrax): the effect of salinity on AQP1 and AQP3 expression. Comp. Biochem. Physiol. 148A, 430-444.

Gradilone, S. A., Garcia, F., Huebert, R. C., Tietz, P. S., Larocca, M. C., Kierbel, A., Carreras, F. I. LaRusso, N. F., and Marinelli, R. A. (2003). Glucagon induces the plasma membrane insertion of functional aquaporin- 8 water channels in isolated rat hepatocytes. Hepatology 37, 1435-1441.

Grosell, M. (2011). Intestinal anion exchange in marine teleosts is involved in osmoregulation and contributes to the oceanic inorganic carbon cycle. Acta Physiol. (Oxf.) 202, 421-434.

Hofmann, K., and Stoffel, W. (1993). TMbase - a database of membrane spanning proteins segments. Biol. Chem. Hoppe Seyler 374, 166.

Ishibashi, K., Hara, S., and Kondo, S. (2009). Aquaporin water channels in mammals. Clin. Exp. Nephrol. 13 107-117.

Kawedia, J. D., Jiang, M., Kulkarni, A., Waechter, H. E., Matlin, K. S., Pauletti, G. M., and Menon, A. G. (2008). The protein kinase A pathway contributes to $\mathrm{Hg}^{2+}$. induced alterations in phosphorylation and subcellular distribution of occludin associated with increased tight junction permeability of salivary epithelial cell monolayers. J. Pharmacol. Exp. Ther. 326, 829-837.

Kim, Y. K., Watanabe, S., Kaneko, T. Do Huh, M., and Park, S. I. (2010). Expression of aquaporins 3, 8 and 10 in the intestines of freshwaterand seawater-acclimated Japanese eels Anguilla japonica. Fish Sci. 76, 695-702.
Kinne-Saffran, E., and Kinne, R. K. (2001). Inhibition by mercuric chloride of $\mathrm{Na}-\mathrm{K}-2 \mathrm{Cl}$ cotransport activity in rectal gland plasma membrane vesicles isolated from Squalus acanthias. Biochim. Biophys. Acta 1510 , 442-451.

Koyama, Y., Yamamoto, T., Tani, T., Nihei, K., Kondo, D., Funaki, H., Yaoita, E., Kawasaki, K., Sato, N., Hatakeyama, K., and Kihara, I. (1999). Expression and localization of aquaporins in rat gastrointestinal tract. Am. J. Physiol. 276, C621C627.

Laforenza, U., Cova, E., Gastaldi, G. Tritto, S., Grazioli, M., LaRusso, N. F., Splinter, P. L., D’Adamo, P., Tosco, M., and Ventura, U. (2005). Aquaporin-8 is involved in water transport in isolated superficial colonocytes from rat proximal colon. J. Nutr. 135, 2329-2336.

Larsen, E. H., and Møbjerg, N. (2006). $\mathrm{Na}^{+}$recirculation and isosmotic transport. J. Membr. Biol. 212, $1-15$.

Liu, K., Nagase, H., Huang, C. G., Calamita, G., and Agre, P. (2006). Purification and functional characterization of aquaporin-8. Biol. Cell 98, 153-161.

Loo, D. D., Wright, E. M., and Zeuthen, T. (2002). Water pumps. J. Physiol. 542, 53-60.

Loretz, C. A. (1995). "Electrophysiology of ion transport in teleost intestinal cells," in Cellular and Molecular Approaches to Fish Ionic Regulation, ed. C. M. Wood and T. J. Shuttleworth (San Diego: Academic Press), 25-56.

Lowry, O. H., Roseborough, N. J., Farr, A. L., and Randall, R. J. (1951) Protein measurement with the folin reagent. J. Biol. Chem. 193, 266-275.

Ma, T. H., and Verkman, A. S. (1999). Aquaporin water channels in gastrointestinal physiology. J. Physiol. 517, 317-326

Ma, T. H., Yang, B. X., and Verkman, A. S. (1997). Cloning of a novel water and urea-permeable aquaporin from mouse expressed strongly in colon, placenta, liver, and heart. Biochem. Biophys. Res. Commun. 240, 324-328. 
MacIver, B., Cutler, C. P., Yin, J., Hill, M. G., Zeidel, M. L., and Hill, W. G. (2009). Expression and functional characterization of four aquaporin water channels from the European eel (Anguilla anguilla). J. Exp. Biol. 212, 2856-2863.

Martinez, A. S., Cutler, C. P., Wilson, G. D., Phillips, C., Hazon, N., and Cramb, G. (2005a). Cloning and expression of three aquaporin homologues from the European eel (Anguilla anguilla): effects of seawater acclimation and cortisol treatment on renal expression. Biol. Cell 97, 615-627.

Martinez, A. S., Cutler, C. P., Wilson, G. D., Phillips, C., Hazon, N., and Cramb, G. (2005b). Regulation of expression of two aquaporin homologs in the intestine of the European eel: effects of seawater acclimation and cortisol treatment. Am. J. Physiol. Regul. Integr. Comp. Physiol. 288, R1733-R1743.

Nedergaard, S., Larsen, E. H., and Ussing, H. H. (1999). Sodium recirculation and isotonic transport in toad small intestine. J. Membr. Biol. 168, 241-251.

Pandey, R. N., Yaganti, S., Coffey, S., Frisbie, J., Alnajjar, K., and Goldstein, D. (2010). Expression and immunolocalization of aquaporins HC-1, -2, and -3 in Cope's gray treefrog, $H y l a$ chrysoscelis. Comp. Biochem. Physiol. 157A, 86-94.

Perrott, M. N., Grierson, C. E., Hazon, N., and Balment, R. J. (1992). Drinking behavior in seawater and fresh-water teleosts, the role of the renin-angiotensin system. Fish Physiol. Biochem. 10, 161-168.

Polakof, S., Álvarez, R., and Soengas, J. L. (2010). Gut glucose metabolism in rainbow trout: implications in glucose homeostasis and glucosensing capacity. Am. J. Physiol. Regul. Integr. Comp. Physiol. 299, R19-R32.
Preston, G. M., Caroll, T. P., Guggino, W. B., and Agre, P. (1992). Appearance of water channels in Xenopus oocytes expressing redcell CHIP28 protein. Science 256, 385-387.

Raldúa, D., Otero, D., Fabra, M., and Cerdà, J. (2008). Differential localization and regulation of two aquaporin-1 homologs in the intestinal epithelia of the marine teleost Sparus aurata. Am. J. Physiol. Regul. Integr. Comp. Physiol. 294, R993-R1003.

Santos, C. R., Estêvão, M. D., Fuentes, J., Cardoso, J. C., Fabra, M., Passos, A. L., Detmers, F. J., Deen, P. M., Cerdà, J., and Power, D. M. (2004). Isolation of a novel aquaglyceroporin from a marine teleost (Sparus auratus): function and tissue distribution. J. Exp. Biol. 207, 1217-1227.

Savage, D. F., and Stroud, R. M. (2007). Structural basis of aquaporin inhibition by mercury. J. Mol. Biol. 368, 607-617.

Smith, H. W. (1932). The absorption and excretion of water and salts by marine teleosts. Q. Rev. Biol. 7, 1-26. Sundell, K., Jutfelt, F., Agustsson, T., Olsen, R.-E., Sandblom, E., Hansen, T., and Björnsson, B. T. (2003). Intestinal transport mechanisms and plasma cortisol levels during normal and out-of-season parrsmolt transformation of Atlantic salmon, Salmo salar. Aquaculture 222, 265-285.

Suzuki, M. (2010). Expression and localization of aquaporin-1 on the apical membrane of enterocytes in the small intestine of bottlenose dolphins. J. Comp. Physiol. B 180, 229-238.

Tingaud-Sequeira, A., Calusinska, M., Finn, R. N., Chauvigne, F., Lozano, J., and Cerdà, J. (2010). The zebrafish genome encodes the largest vertebrate repertoire of functional aquaporins with dual paralogy and substrate specificities similar to mammals. BMC Evol. Biol. 10, 38. doi: 10.1186/1471-2148-10-38

Tingaud-Sequeira, A., Chauvigné, F., Fabra, M., Lozano, J., Raldúa, D., and Cerdà, J. (2008). Structural and functional divergence of two fish aquaporin-1 water channels following teleost-specific gene duplication. BMC Evol. Biol. 8, 259. doi: 10.1186/1471-2148-8-259

Tipsmark, C. K., Sørensen, K. J., and Madsen, S. S. (2010). Aquaporin expression dynamics in osmoregulatory tissues of Atlantic salmon during smoltification and seawater acclimation. J. Exp. Biol. 213, 368-379.

Tritto, S., Gastaldi, G., Zelenin, S., Grazioli, M., Orsenigo, M. N., Ventura, U., Laforenza, U., and Zelenina, M. (2007). Osmotic water permeability of rat intestinal brush border membrane vesicles: involvement of aquaporin-7 and aquaporin-8 and effect of metal ions. Biochem. Cell Biol. 85, 675-684.

Usher, M. L., Talbot, C., and Eddy, F. B. (1991). Intestinal water transport in juvenile Atlantic salmon (Salmo salar L.) during smolting and following transfer to seawater. Comp. Biochem. Physiol. A Comp Physiol. 100A, 813-818.

Valenti, G., Procino, G., Tamma, G., Carmosino, M., and Svelto, M. (2005). Minireview: aquaporin 2 trafficking. Endocrinology 146, 5063-5070.

Veillette, P. A., White, R. J., and Specker, J. L. (1993). Changes in intestinal fluid transport in Atlantic salmon (Salmo salar) during parrsmolt transformation. Fish Physiol. Biochem. 12, 193-202.

Veillette, P. A., White, R.J. and Specker, J. L., and Young, G. (2005). Osmoregulatory physiology of pyloric ceca: regulated and adaptive changes in chinook salmon. J. Exp. Zool. 303A, 608-613.

Wang, X., and Horrisberger, J.-D. (1996). Mercury binding site on
$\mathrm{Na}^{+} / \mathrm{K}^{+}$-ATPase: a cysteine in the first transmembrane segment. Mol. Pharmacol. 50, 687-691.

Xia, X. B., Lin, J. T., and Kinne, R. K. (2003). Binding of phlorizin to the isolated C-terminal extramembranous loop of the $\mathrm{Na}^{+}$/glucose cotransporter assessed by intrinsic tryptophan fluorescence. Biochemistry 42, 6115-6120.

Yool, A. J., Brokl, O. H., Pannabecker, T. L., Dantzler, W. H., and Stamer, W. D. (2002). Tetraethylammonium block of water flux in aquaporin1 channels expressed in kidney thin limbs of Henle's loop and a kidney-derived cell line. BMC Physiol. 2, 4. doi: 10.1186/14726793-2-4

Conflict of Interest Statement: The authors declare that the research was conducted in the absence of any commercial or financial relationships that could be construed as a potential conflict of interest.

Received: 14 June 2011; accepted: 17 August 2011; published online: 07 September 2011.

Citation: Madsen SS, Olesen JH, Bedal $K$, Engelund MB, Velasco-Santamaría YM and Tipsmark CK (2011) Functional characterization of water transport and cellular localization of three aquaporin paralogs in the salmonid intestine. Front. Physio. 2:56. doi: 10.3389/fphys.2011.00056

This article was submitted to Frontiers in Aquatic Physiology, a specialty of Frontiers in Physiology.

Copyright (C) 2011 Madsen, Olesen, Bedal, Engelund, Velasco-Santamaría and Tipsmark. This is an open-access article subject to a non-exclusive license between the authors and Frontiers Media $S A$, which permits use, distribution and reproduction in other forums, provided the original authors and source are credited and other Frontiers conditions are complied with. 